\title{
Modular Hamiltonians for deformed half-spaces and the averaged null energy condition
}

\author{
Thomas Faulkner, Robert G. Leigh, Onkar Parrikar and Huajia Wang \\ Department of Physics, University of Illinois, \\ 1110 W. Green St., Urbana IL 61801-3080, U.S.A. \\ E-mail: tomf@illinois.edu, rgleigh@illinois.edu, \\ parrika2@illinois.edu, rockwhj@illinois.edu
}

ABSTRACT: We study modular Hamiltonians corresponding to the vacuum state for deformed half-spaces in relativistic quantum field theories on $\mathbb{R}^{1, d-1}$. We show that in addition to the usual boost generator, there is a contribution to the modular Hamiltonian at first order in the shape deformation, proportional to the integral of the null components of the stress tensor along the Rindler horizon. We use this fact along with monotonicity of relative entropy to prove the averaged null energy condition in Minkowski space-time. This subsequently gives a new proof of the Hofman-Maldacena bounds on the parameters appearing in CFT three-point functions. Our main technical advance involves adapting newly developed perturbative methods for calculating entanglement entropy to the problem at hand. These methods were recently used to prove certain results on the shape dependence of entanglement in CFTs and here we generalize these results to excited states and real time dynamics. We also discuss the AdS/CFT counterpart of this result, making connection with the recently proposed gravitational dual for modular Hamiltonians in holographic theories.

Keywords: AdS-CFT Correspondence, Field Theories in Higher Dimensions

ARXIV EPRINT: 1605.08072 


\section{Contents}

1 Introduction $\quad 1$

1.1 Setup \& summary of results 3

2 Modular Hamiltonian for deformed half-space $\quad 7$

$\begin{array}{ll}2.1 \text { Reduced density matrix } & 7\end{array}$

2.2 Modular Hamiltonian 9

3 Averaged null energy condition $\quad 15$

$\begin{array}{ll}3.1 \text { Positivity of } \widehat{K}_{A_{0}}-\widehat{K}_{A} & 15\end{array}$

3.2 Computing $\left\langle\widehat{K}_{A_{0}}\right\rangle_{\psi}-\left\langle\widehat{K}_{A}\right\rangle_{\psi} \quad 16$

4 Modular Hamiltonians in AdS/CFT $\quad 17$

5 Discussion $\quad 21$

5.1 Sharpening the argument 22

5.2 Generalizations 23

$\begin{array}{ll}\text { A Cutoff at the entangling surface } & 25\end{array}$

\section{Introduction}

The entanglement structure of states is of great importance in quantum field theory. The most common tool used for studying entanglement structure is the entanglement entropy, namely the Von Neumann entropy of a subregion, and has already provided many important insights. A more fine-grained probe is the modular Hamiltonian, defined as

$$
K_{A}^{\Psi}=-\ln \rho_{A}^{\Psi}
$$

where $\rho_{A}^{\Psi}$ is the reduced density matrix of the state $\Psi$ over the subregion $A$. The modular Hamiltonian is, in general, a complicated, non-local operator and not of much practical use. However, the situation greatly simplifies for the vacuum state in the case of certain special symmetric subregions. For instance, the modular Hamiltonian for a half-space in relativistic quantum field theories takes a very simple form; it is the restriction to the half space of the generator of boosts which preserve the entangling surface [1], and consequently generates a local and geometric modular flow. A similar construction is also possible for spherical subregions in conformal field theories [2], for null slabs in the case where the vacuum state is defined with respect to the generator of null translations on a null hypersurface $[3,4]$ etc. Recently, it has been argued that the modular Hamiltonian for states with classical gravitational duals also takes a simple form $[5,6]$. On the other hand, it is quite non-trivial 
to study the entanglement entropy and modular Hamiltonian for more general (asymmetric) subregions, especially outside the purview of free-field theories or AdS/CFT. Some progress was made in [7] (following previous work in [8-12]), where perturbative techniques were used to study the shape-dependence of entanglement entropy in conformal field theories. In the present paper, we adapt these techniques to study modular Hamiltonians for deformed half-spaces in relativistic (not necessarily conformal) quantum field theories.

The study of shape dependence of entanglement is an important task for several reasons. The entanglement structure of quantum systems is highly constrained by powerful inequalities, such as strong subadditivity of entanglement entropy, positivity and monotonicity of relative entropy, etc. In many situations, these entanglement inequalities further imply fundamental constraints on the properties of quantum field theories. For instance, the strong subadditivity property was used in $[13,14]$ to prove an entropic version of the c-theorem for renormalization group flows in two and three dimensions. Similarly, the properties of relative entropy have been used to prove several interesting results such as the Bekenstein bound [15], the generalized second law for causal horizons [16] and the covariant entropy bound in the context of semi-classical gravity [3, 4]. Entanglement inequalities have also been shown to constrain the bulk geometry in states with classical gravity duals [17-20]. The entanglement inequality which will be relevant for our purpose is that the full modular Hamiltonian for the vacuum state

$$
\widehat{K}_{A}=K_{A}-K_{A^{c}}
$$

(i.e., the difference between the modular Hamiltonian of the subregion $A$ and that of the complementary subregion $A^{c}$ ) satisfies a "monotonicity" property under inclusion [21]. This means that if we shrink the subregion $A$, then the corresponding change in the full modular Hamiltonian $\delta \widehat{K}_{A}$ is a negative semi-definite operator. This property in fact follows from the monotonicity of relative entropy, as was shown in [22]. In the present work, we will show that this monotonicity property of the full modular Hamiltonian along with perturbative results on the shape dependence of the modular Hamiltonian allow us to prove another fundamental constraint, namely the averaged null energy condition (ANEC)

$$
\int_{-\infty}^{\infty} d x^{+}\left\langle T_{++}\left(x^{+}, x^{-}=0, \vec{x}^{i}\right)\right\rangle_{\psi} \geq 0
$$

for excited state in relativistic quantum field theories on Minkowski space-time.

From a classical general relativity point of view, averaged energy conditions (which are weaker than the point-wise null, weak, strong, dominant-energy conditions) were shown to be sufficient for proving a number of interesting results such as standard focussing theorems [23, 24], topological censorship [25], etc. This provides a clear motivation for trying to prove or disprove averaged energy conditions for quantum fields in general spacetimes, given that most point-wise conditions are known to be violated by quantum effects (see for instance, [26]). ${ }^{1}$ In Minkowski space-time, the ANEC has been proven to hold

\footnotetext{
${ }^{1}$ However, there are alternative proposals for point-wise quantum energy conditions. See for example [27-29].
} 
for many special cases such as free scalar and Maxwell fields in general dimension [30-32], arbitrary quantum field theories in $d=2$ with a mass gap and some assumptions on the stress tensor [33], CFTs with classical gravitational duals in general dimension [34], etc. Wall has also argued that the ANEC holds true for free or superrenormalizable field theories in general dimension [16]. In summary, there is substantial evidence so far to suggest that the ANEC is satisfied by generic quantum field theories on Minkowski space-time, but a general proof has been missing hitherto (although see [35] for an argument involving certain assumptions on the OPE of non-local operators) - in this paper, we will partially fill this gap. On the other hand, the ANEC is known to be violated in general curved space-times, but an alternative proposal called the self-consistent achronal ANEC exists in this case see [36-38] and references there-in for further discussion. While this is out of the scope of the present paper, our results can nevertheless be extended to prove the ANEC along static bifurcate Killing horizons even in curved space-times.

There is another motivation for trying to prove the ANEC in Minkowski space-time. In [39], Hofman and Maldacena (HM) showed that in a conformal field theory the validity of the ANEC in a certain class of states created by operator insertions implies bounds on the coefficients appearing in the three-point correlation functions of that CFT. For instance, in $d=4$ they used this to derive a bound on the ratio of central charges

$$
\frac{1}{3} \leq \frac{a}{c} \leq \frac{31}{18}
$$

where $a$ and $c$ are the coefficients of the Euler density term and the Weyl tensor squared term in the conformal anomaly; tighter bounds can be obtained by imposing supersymmetry. While the assumption of the ANEC was considered reasonable, in the original paper no proof was given. Since then, there have been several attempts at a proof of the HM bounds with varying levels of success [35, 40, 41].

In particular, using analytic bootstrap methods the HM bounds were proven for a class of three-point functions in [42], building on the work of [43, 44]. These methods take as an input crossing symmetry and reflection positivity and apply these principles to various four point functions in a light-cone limit to delicately extract the HM bounds. In particular in this guise the HM bounds were related to causality properties of correlation functions in a shockwave background [44]. ${ }^{2}$ In contrast, we will show that the general HM constraints on CFT three-point functions can be extracted directly from the three-point function itself - when the three-point function is interpreted as calculating some modular energy of the CFT in an excited state.

Overall it is satisfying to see the ANEC, and consequently the Hofman-Maldacena bounds, arise as a natural consequence of the fundamental constraints satisfied by the entanglement structure of the vacuum.

\subsection{Setup \& summary of results}

We now outline the calculation we are interested in, and present a brief summary of our results. Consider the density matrix $|\Psi\rangle\langle\Psi|$ corresponding to a pure state defined on the

\footnotetext{
${ }^{2}$ In theories with gravity duals, the HM bounds have also been shown to be related to bulk causality constraints [34, 35, 45, 46].
} 
Cauchy surface $\Sigma$. Let us partition $\Sigma$ into two subregions $A$ and its complement $A^{c}$. For local quantum field theories, we expect the Hilbert space $\mathfrak{h}_{\Sigma}$ to factorize into the tensor product $\mathfrak{h}_{\Sigma}=\mathfrak{h}_{A} \otimes \mathfrak{h}_{A^{c}}$. If this is the case, we can trace over $\mathfrak{h}_{A^{c}}$ to obtain the reduced density matrix

$$
\rho_{A}^{\Psi}=\operatorname{Tr}_{A^{c}}(|\Psi\rangle\langle\Psi|)
$$

which contains all the relevant information pertaining to the subregion $A$. The entanglement entropy between $A$ and $A^{c}$ is defined as the von Neumann entropy of $\rho_{A}^{\Psi}$

$$
S_{\mathrm{EE}}[\Psi, A]=-\operatorname{Tr}_{A}\left(\rho_{A}^{\Psi} \ln \rho_{A}^{\Psi}\right) .
$$

In this context, the boundary $\partial A$ of $A$ is referred to as the entangling surface. The modular Hamiltonian (also known as the entanglement Hamiltonian) $K_{A}^{\Psi}$ is defined as

$$
K_{A}^{\Psi}=-\ln \rho_{A}^{\Psi}
$$

Similarly, we can also define the modular Hamiltonian corresponding to the region $A^{c}$, which we denote $K_{A^{c}}^{\Psi}$. We can combine $K_{A}^{\Psi}$ and $K_{A^{c}}^{\Psi}$ into another useful operator:

$$
\widehat{K}_{A}^{\Psi}=K_{A}^{\Psi} \otimes \mathbb{1}_{A^{c}}-\mathbb{1}_{A} \otimes K_{A^{c}}^{\Psi}
$$

which we will refer to as the full modular Hamiltonian.

In this paper, we will primarily study the operators $K_{A}, K_{A^{c}}$ and $\widehat{K}_{A}$ for the vacuum state of a relativistic quantum field theory (as such we drop the label $\Psi$ from now on), with the region $A$ being a slightly deformed half-space. To specify the geometry in more detail, let us pick global coordinates $x^{\mu}=\left(x^{0}, x^{1}, \cdots, x^{d-1}\right)=\left(x^{0}, \mathbf{x}\right)$ on $\mathbb{R}^{1, d-1}$, where $x^{0}$ is the time coordinate, and $\mathbf{x}$ denotes spatial coordinates. Pick the Cauchy surface $\Sigma$ given by $x^{0}=0$, and consider the half space $A_{0}$ given by

$$
A_{0}=\left\{x^{\mu} \in \mathbb{R}^{1, d-1} \mid x^{0}=0, x^{1}>0\right\} .
$$

The vacuum modular Hamiltonian for the half space takes a particularly simple form [1]

$$
K_{A_{0}}=2 \pi \int_{A_{0}} d^{d-1} \mathbf{x} x^{1} T_{00}(0, \mathbf{x})+\text { constant }
$$

i.e., it is the generator of boosts which preserve the entangling surface restricted to the region $A_{0}$, a result known as the Bisognano-Wichmann theorem [1]. Correspondingly, the full modular Hamiltonian is given by the full boost generator

$$
\widehat{K}_{A_{0}}=2 \pi \int_{\Sigma} d^{d-1} \mathbf{x} x^{1} T_{00}(0, \mathbf{x})
$$

Note that $\widehat{K}_{A_{0}}$ is a conserved charge, and as such annihilates the vacuum $\widehat{K}_{A_{0}}|0\rangle=0$. (This later property is true for more general regions as well.)

One can consider a small deformation of the region $A_{0}$ to

$$
A=\left\{x^{\mu} \in \mathbb{R}^{1, d-1} \mid x^{0}=0, x^{1}>\zeta(\vec{x})\right\}
$$




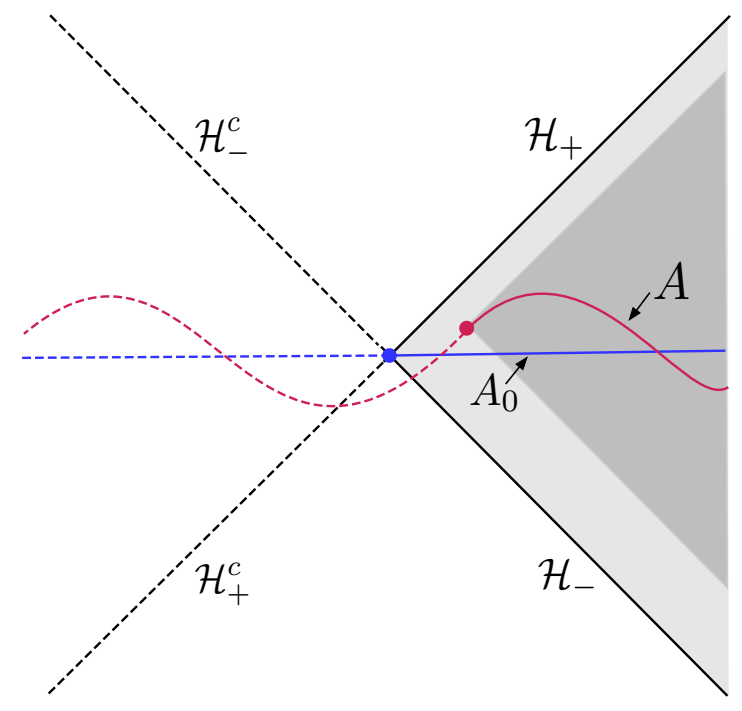

Figure 1. We deform the half space $A_{0}$ (solid blue line) inwards into the region $A$ (solid red line), such that $\mathcal{D}(A)$ (darker shaded region) is contained inside $\mathcal{D}\left(A_{0}\right)$ (lighter shaded region). Also shown are the Rindler horizons $\mathcal{H}_{ \pm}$corresponding to the regions $A_{0}$ and $A_{0}^{c}$. (The transverse directions $\vec{x}$ are implicit.)

where $\zeta(\vec{x})$ is a smooth function of the $(d-2)$ transverse spatial coordinates (parametrizing the entangling surface), collectively denoted by $\vec{x}=\left(x^{2}, \cdots, x^{d-1}\right)$. The deformation is special in that it is restricted within the original Cauchy surface. We can generalize this to also include time-like deformations (see figure 1)

$$
A=\left\{\tilde{x}^{\mu} \in \mathbb{R}^{1, d-1} \mid \tilde{x}^{0}=\zeta^{0}\left(x^{1}, \vec{x}\right), \tilde{x}^{1}=x^{1}+\zeta^{1}\left(x^{1}, \vec{x}\right), x^{1}>0\right\} .
$$

We identify the infinitesimal $\zeta^{\mu}$ as the deformation vector field and pick $\zeta^{\mu}$ to point inward, i.e. $\mathcal{D}(A) \subset \mathcal{D}\left(A_{0}\right)$ (where $\mathcal{D}$ denotes the domain of dependence).

Our primary results in this paper are as follows:

(i) We will first show that the modular Hamiltonian $K_{A}$, up to first order in the shape deformation, is given by

$$
U K_{A} U^{\dagger}=K_{A_{0}}-2 \pi \int_{\mathcal{H}_{+}} \zeta^{+} T_{++}+2 \pi \int_{\mathcal{H}_{-}} \zeta^{-} T_{--}+2 \pi \int_{A_{0}} \zeta^{\nu}\left[K_{A_{0}}, T_{0 \nu}\right]+O\left(\zeta^{2}\right)
$$

where $U: \mathfrak{h}_{A} \rightarrow \mathfrak{h}_{A_{0}}$ is a unitary transformation the details of which we will specify later, $\mathcal{H}_{ \pm}$are the future and past Rindler horizons of $\mathcal{D}\left(A_{0}\right)$ shown in figure 1 , and $\zeta^{ \pm}$ appearing in the second and third terms above are components of the vector field $\zeta$ on the entangling surface in light-cone coordinates $x^{ \pm}=x^{0} \pm x^{1}$. Similar expressions can also be written for $K_{A^{c}}$ and $\widehat{K}_{A}$.

(ii) We will then consider the expectation value $\left\langle\psi\left|\widehat{K}_{A}\right| \psi\right\rangle$ in states of the form

$$
\left|\psi_{\alpha}\right\rangle=e^{-\tau H} \mathcal{O}_{\alpha}(0, \mathbf{x})|0\rangle
$$


and linear combinations thereof, where $H$ is the Hamiltonian and $\mathcal{O}_{\alpha}$ is an arbitrary local operator whose quantum numbers (dimension, spin etc.) are collectively denoted by $\alpha .{ }^{3}$ The factor of $e^{-\tau H}$ is added to make these states normalizable. In a CFT this class of states is a basis for the entire Hilbert space, via the state-operator mapping. For a general QFT similar statements should hold. In fact, there is no obstruction to generalizing our argument to include states created by many local and even non-local operators inserted throughout the lower half Euclidean plane. Further, we could also insert the operators in real time. In the interest of simplifying our presentation we choose to represent our state via a single operator insertion on the Euclidean section, although we expect all our conclusions to go through even in the more general case.

We then show that equation (1.14), along with the positivity of the operator $\widehat{K}_{A_{0}}-\widehat{K}_{A}$ (i.e. monotonicity under inclusion, which recall follows from the monotonicity of relative entropy) implies the averaged null energy condition (ANEC)

$$
\int_{-\infty}^{\infty} d x^{+}\left\langle T_{++}\left(x^{+}, x^{-}=0, \vec{x}\right)\right\rangle_{\psi} \geq 0
$$

As discussed in the introduction, the Hofman-Maldacena bounds on CFT threepoint functions were derived assuming the ANEC; so this completes the proof of these bounds. It should perhaps be mentioned that the specific states considered in deriving the HM bounds in [39] were created by inserting an approximately local operator in real time, with an approximately specified four-momentum. Given our remarks below equation (1.15), our derivation of the ANEC also applies to these states.

(iii) Finally, we also discuss the (vacuum) full modular Hamiltonian for deformed halfspaces in CFTs with classical gravity duals, which allows us to make contact with the recent proposal by Jafferis-Lewkowyzc-Maldacena-Suh (JLMS) [6] for the holographic dual to the modular Hamiltonian.

At this point we should mention that in continuum quantum field theory there are significant ultraviolet (UV) issues associated with the definition of the reduced density matrix for a region, often resulting in divergences for entanglement entropy and modular energy which are local to the entangling surface. These issues and associated divergences are however not present for quantities like the relative entropy, and the full modular Hamiltonian [47, 48]. Since this is ultimately what we are interested in, and in the interest of simplicity of presentation, we will for the most part suppress the need for a UV cutoff at the entangling surface. Indeed the answers we will find will be finite, partly justifying this approach. For further discussion on how to include such a UV cutoff in our calculation, see appendix A, where we will argue for the irrelevance of the details of such a cutoff beyond its existence.

\footnotetext{
${ }^{3}$ In the case of tensor operators, we contract them with appropriate polarizations, for instance $\mathcal{O}_{\alpha}(x)=$ $\epsilon_{\mu_{1} \mu_{2} \cdots \mu_{s}} J^{\mu_{1} \mu_{2} \cdots \mu_{s}}(x)$
} 


\section{Modular Hamiltonian for deformed half-space}

In this section, we give an explicit formula for the modular Hamiltonian $K_{A}$ of the vacuum state over a deformed half-space, to first order in the shape deformation.

\subsection{Reduced density matrix}

The vacuum state in a relativistic quantum field theory can be constructed by performing the Euclidean path-integral over the region $x_{E}^{0}<0$, where $x_{E}^{0}$ is Euclidean time. In the interest of generality, let us instead consider a more general state rather than the vacuum ${ }^{4}$

$$
|\psi\rangle=\sum_{\alpha} c_{\alpha}\left|\psi_{\alpha}\right\rangle=e^{-\tau H} \sum_{\alpha} c_{\alpha} \mathcal{O}_{\alpha}(0, \mathbf{x})|0\rangle, \quad \cdots \quad(\tau>0)
$$

This state can be constructed similarly as a sum over path-integrals, but with the operator $\mathcal{O}_{\alpha}$ inserted at $x_{E}^{0}=-\tau$ in the term proportional to $c_{\alpha}$. The reduced density matrix corresponding to $|\psi\rangle$, associated with the undeformed half-space $A_{0}$ is constructed as a Euclidean path integral with specified field configurations above $\left(x_{E}^{0} \rightarrow 0^{+}, x^{1}>0\right)$ and below $\left(x_{E}^{0} \rightarrow 0^{-}, x^{1}>0\right)$ the region $A_{0}$ (see figure 2 )

$$
\left\langle\alpha_{0}\left|\rho_{A_{0}, \eta}^{\psi}\right| \beta_{0}\right\rangle=\mathcal{N}_{A_{0}, \eta}^{-1} \int_{\phi^{-}=\alpha_{0}}^{\phi^{+}=\beta_{0}}[D \phi]_{\eta} \sum_{\alpha} \sum_{\alpha^{\prime}} c_{\alpha}^{*} c_{\alpha^{\prime}} \mathcal{O}_{\alpha}^{\dagger}(\tau, \mathbf{x}) \mathcal{O}_{\alpha^{\prime}}(-\tau, \mathbf{x}) e^{-S[\eta, \phi(x)]}
$$

where we have collectively denoted all the fields integrated over in the path integral as $\phi$, and $\left.\left|\alpha_{0}\right\rangle, \beta_{0}\right\rangle \in \mathfrak{h}_{A_{0}}$ are eigenstates of the field operator $\phi$ restricted to $A_{0}$. The prefactor $\mathcal{N}_{A_{0}, \eta}^{-1}$ is added to ensure the normalization of the density matrix, i.e. $\operatorname{Tr}_{A_{0}} \rho_{A_{0}, \eta}^{\psi}=1$. We have explicitly displayed the dependence of the reduced density matrix on the metric $\eta_{\mu \nu}{ }^{5}$ through the path integral measure (which we assume is diffeomorphism invariant), the action and the normalization.

For convenience, we will henceforth use the notation (inside path-integrals)

$$
X=\sum_{\alpha} \sum_{\alpha^{\prime}} c_{\alpha}^{*} c_{\alpha^{\prime}} \mathcal{O}_{\alpha}^{\dagger}(\tau, \mathbf{x}) \mathcal{O}_{\alpha^{\prime}}(-\tau, \mathbf{x})
$$

Now consider the reduced density matrix over the deformed region $A$. This can be constructed by a similar Euclidean path integral with specified field configurations above and below $A$, and with a real-time fold around $x_{E}^{0}=0$ in the case of time-like deformations $\left(\zeta^{0} \neq 0\right)$. We can deal with this path integral by performing a diffeomorphism $f: x^{\mu} \rightarrow$ $x^{\mu}-\zeta^{\mu}$, which maps $A$ to $A_{0}$. We can take $\zeta^{\mu}$ to be non-vanishing (corresponding to nontrivial $f$ ) only within a small region $\left|x_{E}^{0}\right|<\ell$ (for some $\ell \ll \tau$, but much larger than the cutoff). Of course, such a diffeomorphism has a non-trivial action on the background metric

$$
g=\left(f^{-1}\right)^{*} \eta
$$

\footnotetext{
${ }^{4}$ Later, we will also need to compute the expectation value $\left\langle K_{A}\right\rangle_{\psi}=\operatorname{Tr}_{A}\left(\rho_{A}^{\psi} K_{A},\right)$ in the excited state $|\psi\rangle$; so we derive the reduced density matrix $\rho_{A}^{\psi}$ along the way while setting up the calculation for $K_{A}$.

${ }^{5}$ Here by $\eta$ we are denoting the metric in real time. Of course the corresponding metric on the Euclidean section which is used in constructing the Euclidean path integral is $\delta_{\mu \nu}$.
} 


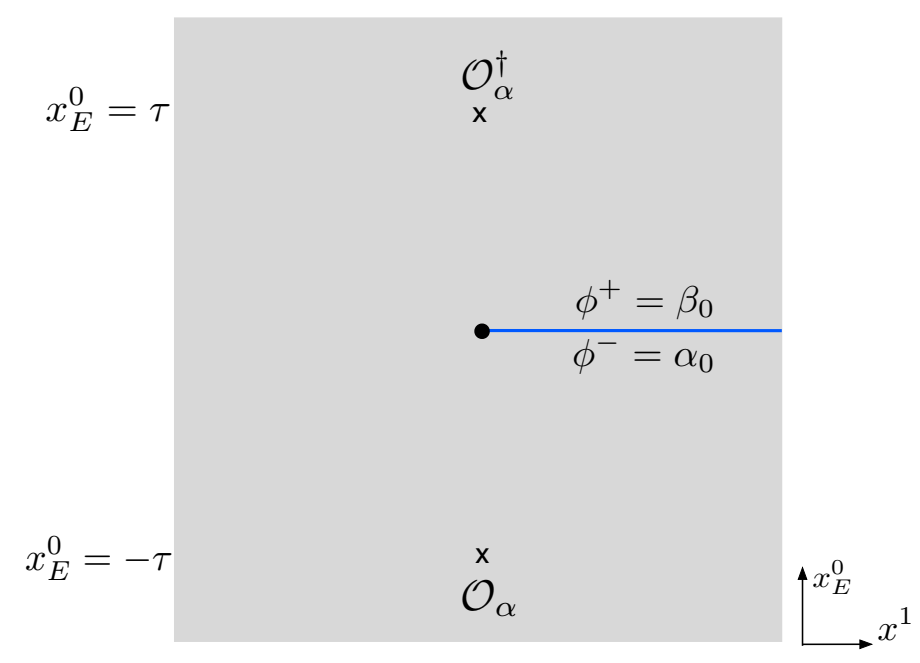

Figure 2. The path integral construction for matrix elements of the reduced density matrix for the state $\left|\psi_{\alpha}\right\rangle$, over the original half space $A_{0}$ (solid blue line). The operator insertions are marked at $x_{E}^{0}= \pm \tau$. The black dot is the entangling surface (with transverse directions $\vec{x}$ implicit).

where $*$ denotes the pullback. We claim that the reduced density matrix over $A$ (with the metric $\eta$ ) is given by

$$
\rho_{A, \eta}^{\psi}=U^{\dagger} \rho_{A_{0}, g}^{\psi} U
$$

where $U$ is a unitary transformation, and $\rho_{A_{0}, g}^{\psi}$ is the reduced density matrix over the undeformed half-space, but with the deformed metric $g$.

We now give a quick formal proof of this claim. If we denote the eigenstates of $\phi$ restricted to $A$ by $|\alpha\rangle,|\beta\rangle \cdots \in \mathfrak{h}_{A}$, then we can construct a unitary ${ }^{6}$ operator $U: \mathfrak{h}_{A} \rightarrow \mathfrak{h}_{A_{0}}$ given by

$$
U=\int[D \alpha]_{\eta}\left|\left(f^{-1}\right)^{*} \alpha\right\rangle\langle\alpha|
$$

Then the claim (2.5) can be checked explicitly by a series of manipulations on the pathintegral definitions of the above density matrices [49]

$$
\begin{aligned}
\left\langle\alpha\left|\rho_{A, \eta}^{\psi}\right| \beta\right\rangle & =\mathcal{N}_{A, \eta}^{-1} \int_{\phi^{-}=\alpha}^{\phi^{+}=\beta}[D \phi]_{\eta} X e^{-S[\eta, \phi]} \\
& =\mathcal{N}_{A, \eta}^{-1} \int_{\left(f^{*} \tilde{\phi}\right)^{-}=\alpha}^{\left(f^{*} \tilde{\phi}\right)^{+}=\beta}\left[D\left(f^{*} \tilde{\phi}\right)\right]_{\eta} X e^{-S\left[\eta,\left(f^{*} \tilde{\phi}\right)\right]} \\
& =\mathcal{N}_{A_{0}, g}^{-1} \int_{\tilde{\phi}^{-}=\left(f^{-1}\right)^{*} \alpha}^{\tilde{\phi}^{+}=\left(f^{-1}\right)^{*} \beta}[D \tilde{\phi}]_{g} X e^{-S[g, \tilde{\phi}]} \\
& =\left\langle\left(f^{-1}\right)^{*} \alpha\left|\rho_{A_{0}, g}^{\psi}\right|\left(f^{-1}\right)^{*} \beta\right\rangle \\
& =\left\langle\alpha\left|U^{\dagger} \rho_{A_{0}, g}^{\psi} U\right| \beta\right\rangle .
\end{aligned}
$$

\footnotetext{
${ }^{6}$ The unitarity follows from the diffeomorphism invariance of the measure: $\left(f^{*}\right)^{*}[D \alpha]_{\eta} \equiv\left[D\left(f^{*} \alpha_{0}\right)\right]_{\eta}=$ $\left[D \alpha_{0}\right]_{g}$.
} 
The first equality follows from the definition of $\rho_{A, \eta}^{\psi}$, the second equality is obtained by changing variables $\phi=f^{*} \tilde{\phi}$ inside the path integral, while the third equality follows from the assumption that the measure is diffeomorphism invariant. We have throughout used the fact that the operator insertions (denoted by $X$, following the definition (2.3)) are away from the region where the diffeomorphism $f$ has non-trivial support, and so $f$ acts trivially on these operators.

In the case where $f$ is an infinitesimal diffeomorphism, we can obtain a perturbative formula for $\rho_{A_{0}, g}^{\psi}$. Writing the deformed metric on the Euclidean section as

$$
g_{\mu \nu}=\delta_{\mu \nu}+2 \partial_{(\mu} \zeta_{\nu)}+O\left(\zeta^{2}\right)
$$

where $\zeta$ is appropriately Wick rotated to Euclidean space, we obtain

$$
U \rho_{A, \eta}^{\psi} U^{\dagger}=\rho_{A_{0}, \eta}^{\psi}+\frac{1}{2} \int d^{d} x \delta g_{\mu \nu}(x) \rho_{A_{0}, \eta}\left\{\frac{\mathcal{T}\left(T^{\mu \nu}(x) X\right)}{\langle X\rangle}-\frac{\left\langle T^{\mu \nu}(x) X\right\rangle X}{\langle X\rangle^{2}}\right\}+O\left(\zeta^{2}\right)
$$

where $\delta g_{\mu \nu}=2 \partial_{(\mu} \zeta_{\nu)}$, and $\mathcal{T}$ is the angular-ordering operator: if $\theta \in(0,2 \pi)$ is the angular coordinate in the $\left(x_{E}^{0}, x^{1}\right)$ plane, then

$$
\mathcal{T}\left(\mathcal{O}_{a}\left(\theta_{a}\right) \mathcal{O}_{b}\left(\theta_{b}\right)\right)=\mathcal{O}_{a}\left(\theta_{a}\right) \mathcal{O}_{b}\left(\theta_{b}\right) H\left(\theta_{a}-\theta_{b}\right)+\mathcal{O}_{b}\left(\theta_{b}\right) \mathcal{O}_{a}\left(\theta_{a}\right) H\left(\theta_{b}-\theta_{a}\right)
$$

where $H$ is the Heaviside step function. For the special case $|\psi\rangle=|0\rangle$, we then obtain

$$
U \rho_{A, \eta} U^{\dagger}=\rho_{A_{0}, \eta}+\frac{1}{2} \int d^{d} x \delta g_{\mu \nu}(x) \rho_{A_{0}, \eta}\left(T^{\mu \nu}(x)-\left\langle T^{\mu \nu}(x)\right\rangle\right)+O\left(\zeta^{2}\right)
$$

\subsection{Modular Hamiltonian}

We are now in a position to construct the modular Hamiltonian over the deformed halfspace for the vacuum state

$$
K_{A, \eta} \equiv-\ln \rho_{A, \eta}=-U^{\dagger}\left(\ln \rho_{A_{0}, g}\right) U=U^{\dagger} K_{A_{0}, g} U
$$

In order to perturbatively expand the right hand side in powers of $\zeta$, we use the resolvent trick

$$
-\ln \rho_{A_{0}, g}=\int_{0}^{\infty} d \lambda\left(\frac{1}{\rho_{A_{0}, g}+\lambda}-\frac{1}{1+\lambda}\right)
$$

which together with equation (2.11) gives

$$
\begin{aligned}
K_{A_{0}, g} & =K_{A_{0}, \eta}+\delta_{\zeta} K_{A_{0}}+O\left(\zeta^{2}\right) \\
\delta_{\zeta} K_{A_{0}} & =-\frac{1}{2} \int_{0}^{\infty} d \lambda \int d^{d} x \delta g_{\mu \nu}(x) \rho_{A_{0}, \eta} \frac{1}{\rho_{A_{0}, \eta}+\lambda}: T^{\mu \nu}:(x) \frac{1}{\rho_{A_{0}, \eta}+\lambda}
\end{aligned}
$$

where we have defined

$$
: T^{\mu \nu}:(x)=T^{\mu \nu}(x)-\left\langle T^{\mu \nu}(x)\right\rangle
$$

In the interest of simplifying notation, we will henceforth drop the explicit reference to the Minkowski metric on $\rho_{A_{0}, \eta}$, and simply refer to it as $\rho_{A_{0}}$. It is possible to perform 


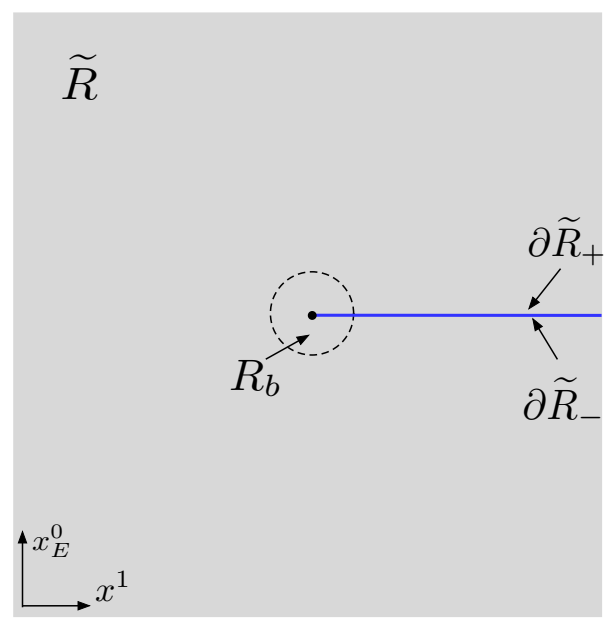

Figure 3. We split the region of integration into two parts: the region inside the dotted line is $R_{b}$, and the region outside is $\widetilde{R}$. Also shown is the brach-cut $\partial \widetilde{R}_{ \pm}$.

the $\lambda$ integral by going to the spectral representation (for details see $[7,12]$, where similar calculations were performed). The result is

$$
\delta_{\zeta} K_{A_{0}}=\frac{1}{2} \int_{-\infty}^{\infty} d s \frac{1}{4 \sinh ^{2}\left(\frac{s+i \epsilon}{2}\right)} \int d^{d} x \delta g^{\mu \nu}(x) \rho_{A_{0}}^{-i s / 2 \pi}: T_{\mu \nu}:(x) \rho_{A_{0}}^{i s / 2 \pi}
$$

Since the operator $\rho_{A_{0}}^{i s / 2 \pi}$ generates modular evolution in Rindler time $s$, we see that the stress tensor is effectively liberated from the Euclidean section and inserted in real time.

We now artificially split the integration region over which the stress tensor is inserted into two parts: a small solid cylinder $R_{b}$ of radius $b$ around the entangling surface, and its complement $\widetilde{R}$. We will later show that the contribution from inside the cylindrical neighborhood vanishes in the limit $b \rightarrow 0$. The region of integration is thus $R=R_{b} \cup \widetilde{R}$ where we should remember that $R$ contains a branch cut along the surface $A_{0}$. We now write $\delta g_{\mu \nu}=\partial_{\mu} \zeta_{\nu}+\partial_{\nu} \zeta_{\mu}$ and integrate by parts on the region $\widetilde{R}$

$$
\delta_{\zeta} K_{A_{0}}=\int_{-\infty}^{\infty} d s \frac{1}{4 \sinh ^{2}\left(\frac{s+i \epsilon}{2}\right)} \rho_{A_{0}}^{-i s / 2 \pi}\left(-\int_{\widetilde{R}}\left(\partial^{\mu} T_{\mu \nu}\right) \zeta^{\nu}+\int_{\partial \widetilde{R}}: T_{\mu \nu}: \zeta^{\nu} d \Sigma^{\mu}\right) \rho_{A_{0}}^{i s / 2 \pi}+\delta_{\zeta} K_{b}
$$

The first term involves the divergence of the stress tensor; in the absence of other operator insertions in the region where $\zeta$ has support, we can drop this term. (Indeed, expectation values in states of the form (1.15) which we will be interested in have precisely this property, since $\zeta$ has no support at the location of the operators $\mathcal{O}_{\alpha}$.) The second term is integrated over $\partial \widetilde{R}=\partial R_{b} \cup \partial \widetilde{R}_{+} \cup \partial \widetilde{R}_{-}$and gets two types of contributions: (i) from the boundary $\partial R_{b}$ of the hole of radius $b$, which we refer to as the imaginary cutoff surface, ${ }^{7}$ and (ii) from $\partial \widetilde{R}_{ \pm}$above and below the region $A_{0}$, which we will refer to as the branch cut (see figure 3). Finally $\delta_{\zeta} K_{b}$ represents the contribution (iii) from inside the cylinder $R_{b}$.

\footnotetext{
${ }^{7}$ Not to be confused with the UV cutoff surface that we discuss in appendix A.
} 
(i) Imaginary cutoff surface: let us first deal with the term supported on the surface $\partial R_{b}$. It is convenient to switch to complex coordinates

$$
z=x^{1}-i x_{E}^{0}, \quad \bar{z}=-\left(x^{1}+i x_{E}^{0}\right) .
$$

In these coordinates, we find

$$
\begin{aligned}
\rho_{A_{0}}^{-i s / 2 \pi}\left(\zeta^{\mu} n^{\nu} T_{\mu \nu}(x)\right) \rho_{A_{0}}^{i s / 2 \pi}=\left(-e^{2 s-i \theta} T_{z z}\left(x_{s}\right)+T_{z \bar{z}}\left(x_{s}\right) e^{i \theta}\right) \zeta^{z} \\
+\left(-T_{z \bar{z}}\left(x_{s}\right) e^{-i \theta}+e^{-2 s+i \theta} T_{\bar{z} \bar{z}}\left(x_{s}\right)\right) \zeta^{\bar{z}}
\end{aligned}
$$

where,

$$
x_{s}^{\mu}=(b \sin (\theta+i s), b \cos (\theta+i s), \vec{x})
$$

Further, $n^{\nu}$ is the (inward pointing) unit normal to $\partial R_{b}$

$$
n=e^{i \theta} \partial_{\bar{z}}-e^{-i \theta} \partial_{z} .
$$

and $\zeta^{z}$ and $\zeta^{\bar{z}}$ are the components of the vector field $\zeta$ close to the entangling surface in holomorphic coordinates

$$
\zeta=\zeta^{z} \partial_{z}+\zeta^{\bar{z}} \partial_{\bar{z}}
$$

We now proceed by shifting the $s$ integration contour $s \rightarrow s+i \theta$ in order to remove the $\theta$ dependence from the stress tensor. We do this after switching the order of integration so that the $s$ integral comes before the $\theta$ integral. This step assumes analyticity in the complex $s$ plane and that the contributions from $s \rightarrow \pm \infty$ vanish, which can be justified in a spectral representation of (2.18). This gives

$$
\begin{aligned}
\left.\delta_{\zeta} K_{A_{0}}\right|_{\partial R_{b}}=-b & \int d^{d-2} \vec{x} \int_{0}^{2 \pi} d \theta \int_{-\infty}^{\infty} d s \frac{1}{4 \sinh ^{2}\left(\frac{s+i \theta}{2}\right)} \\
& \times\left(\left(e^{2 s} T_{z z}-: T_{z \bar{z}}:\right) \zeta^{z} e^{i \theta}+\left(: T_{z \bar{z}}:-e^{-2 s} T_{\bar{z} \bar{z}}\right) \zeta^{\bar{z}} e^{-i \theta}\right)
\end{aligned}
$$

where now these stress-tensors are evaluated at $x_{E}^{0}=i b \sinh (s), x^{1}=b \cosh (s)$. We can now perform the $\theta$ integral using

$$
\int_{0}^{2 \pi} d \theta \frac{1}{4 \sinh ^{2}\left(\frac{s+i \theta}{2}\right)} e^{ \pm i \theta}=2 \pi e^{\mp s} \Theta( \pm s)-2 \pi \delta(s)
$$

The delta function term above can be dropped since this term does not contribute in the limit $b \rightarrow 0 .{ }^{8}$ So we get

$$
\begin{aligned}
\left.\delta_{\zeta} K_{A_{0}}\right|_{\partial R_{b}}=2 \pi b \int d^{d-2} \vec{x}\left(-\int_{0}^{\infty} d s\left(e^{s} T_{z z}-e^{-s}: T_{z \bar{z}}:\right) \zeta^{z}\right. \\
\left.\quad+\int_{-\infty}^{0} d s\left(e^{-s} T_{\bar{z} \bar{z}}-e^{s}: T_{z \bar{z}}:\right) \zeta^{\bar{z}}\right)
\end{aligned}
$$

${ }^{8}$ Actually, rather than drop this term, let us add it to a stack:

$$
\text { Stack }=2 \pi b \int d^{d-2} \vec{x} T_{1 \mu} \zeta^{\mu} .
$$

We will update Stack everytime we find a term of this type in our calculation. 
Naively, it might seem that all the terms on the right hand side vanish in the $b \rightarrow 0$ limit. In fact, the terms involving $T_{z \bar{z}}$ do indeed vanish in this limit. ${ }^{9}$ However, the terms involving $T_{z z}$ and $T_{\bar{z} \bar{z}}$ get an enhancement from the $s$ integral, coming from the $s \sim-\ln b$ and $s \sim \ln b$ limits respectively. Taking the limit $b \rightarrow 0$ and Wick rotating the vector field back to real time, i.e. $\zeta^{z} \rightarrow \zeta^{+}$and $\zeta^{\bar{z}} \rightarrow \zeta^{-}$(where the light-cone coordinates are defined as $x^{ \pm}=x^{0} \pm x^{1}$ ), we obtain

$$
\begin{aligned}
\left.\delta_{\zeta} K_{A_{0}}\right|_{\partial R_{b}}=2 \pi \int d^{d-2} \vec{x}\left(-\int_{0}^{\infty}\right. & d x^{+} \zeta^{+} T_{++}\left(x^{+}, x^{-}=0, \vec{x}\right) \\
& \left.+\int_{-\infty}^{0} d x^{-} \zeta^{-} T_{--}\left(x^{+}=0, x^{-}, \vec{x}\right)\right)
\end{aligned}
$$

where note that the first term on the right hand side is integrated over the future Rindler horizon $\mathcal{H}_{+}$, while the second term is integrated over the past Rindler horizon $\mathcal{H}_{-}$, shown in figure 1 .

(ii) Branch cut: now we come to the second remaining term supported over $\partial \widetilde{R}_{+} \cup \partial \widetilde{R}_{-}$. Once again, deforming the $s$ contours to get rid of the $\theta$ dependence from the stress tensors, we obtain

$$
\begin{aligned}
\left.\delta_{\zeta} K_{A_{0}}\right|_{\partial \widetilde{R}_{ \pm}}=\int d^{d-2} \vec{x} \int_{b}^{\infty} d x^{1} \int_{-\infty}^{\infty} d s \\
\quad \times\left(-\frac{1}{4 \sinh ^{2}\left(\frac{s+i \epsilon}{2}\right)}+\frac{1}{4 \sinh ^{2}\left(\frac{s-i \epsilon}{2}\right)}\right) t^{\mu} \zeta^{\nu} \rho_{A_{0}}^{-i s}: T_{\mu \nu}: \rho_{A_{0}}^{i s}
\end{aligned}
$$

where $t=\partial_{x_{E}^{0}}$, and the stress tensor is evaluated on the region $A_{0}$, i.e. $T_{\mu \nu} \equiv$ $T_{\mu \nu}\left(x_{E}^{0}=0, x^{1}, \vec{x}\right)$ above. The first term inside the brackets comes from $\partial \widetilde{R}_{+}$while the second term comes from $\partial \widetilde{R}_{-}$(after the contour deformation $s \rightarrow s+2 \pi-2 \epsilon$ ). It is clear from equation (2.28) that the $s$ integral precisely picks out the double-pole at $s=0$. A straightforward application of the residue theorem gives

$$
\left.\delta_{\zeta} K_{A_{0}}\right|_{\partial \widetilde{R}_{ \pm}}=2 \pi \int d^{d-2} \vec{x} \int_{b}^{\infty} d x^{1} t^{\mu} \zeta^{\nu}\left[T_{\mu \nu}\left(0, x^{1}, \vec{x}\right), K_{A_{0}}\right]
$$

(iii) Inside the hole: we can follow the same methods as in (i). Pick coordinates close to the entangling surface such that:

$$
d s^{2}=d r^{2}+r^{2} d \theta^{2}+d \vec{x}^{2} \rightarrow d r^{2}-r^{2} d s^{2}+d \vec{x}^{2}
$$

where we have also shown the Wick rotated Rindler coordinates. The hole region $R_{b}$ corresponds to $r<b$ and since we are again working in a region close to the

\footnotetext{
${ }^{9} \mathrm{By}$ this we mean that the $T_{z \bar{z}}$ terms do not contribute to matrix elements in the class of states (1.15) which are of interest here. On the other hand, if we were to evaluate matrix elements in Rindler eigenstates we would find potential divergences in this limit. Note that since we used a spectral representation for $\rho_{A_{0}}$ at an intermediate stage, we were exactly evaluating this in Rindler eigenstates. So the order in which this limit is taken is a somewhat delicate issue which is best ignored on a first pass. In appendix A we confront this issue explicitly.
} 
entangling surface we can take the diffeomorphism at leading order to be independent of $r$. After shifting the integration contour $s \rightarrow s+i \theta$ and Wick rotating the $\zeta$ vector field we have:

$$
\rho_{A_{0}}^{\frac{-i s+\theta}{2 \pi}} T_{\mu \nu}(x) \partial^{\mu} \zeta^{\nu} \rho_{A_{0}}^{\frac{i s-\theta}{2 \pi}}=e^{i \theta+s} T_{i+}\left(x^{+}, x^{-}, \vec{x}\right) \partial_{i} \zeta^{+}+e^{-i \theta-s} T_{i-}\left(x^{+}, x^{-}, \vec{x}\right) \partial_{i} \zeta^{-}
$$

The light-like coordinates where the stress tensor on the right hand side above is located are $x^{ \pm}= \pm r e^{ \pm s}$. We still have to integrate (2.31) over:

$$
\int d^{d} x \int d s \frac{1}{4 \sinh ^{2}\left(\frac{s+i \theta}{2}\right)} \ldots=\int d^{d-2} \vec{x} \int_{r<b} d r r \oint d \theta \int_{-\infty}^{\infty} d s \frac{1}{4 \sinh ^{2}\left(\frac{s+i \theta}{2}\right)} \ldots
$$

But at this point the $\theta$ dependence is the same as in (2.25) and we can again do the $\theta$ integral. After ignoring the $\delta(s)$ contribution which vanishes in the limit $b \rightarrow 0,{ }^{10}$ this has exactly the effect of switching the angular integral in the Euclidean calculation to a real time integral localized near the Rindler horizon: $0<r<b$ and $-\infty<s<\infty$ (see figure 4). The integrand is the stress tensor coupled to a real time diffeomorphism of the metric

$$
\delta_{\zeta} K_{b}=2 \pi \int_{0<r<b} d^{d} x T_{\mu \nu} \partial^{\mu} \widetilde{\zeta}^{\nu}
$$

for the following vector field:

$$
\widetilde{\zeta}=\Theta\left(x^{0}\right) \zeta^{+} \partial_{+}+\Theta\left(-x^{0}\right) \zeta^{-} \partial_{-}
$$

We have again ignored a contribution localized at $x^{0}=0$, coming from the derivative of the step functions above, which vanishes in the limit $b \rightarrow 0 .{ }^{11}$

It is not hard to see that (2.33) should vanish in the limit $b \rightarrow 0$. However it is somewhat enlightening to go another route and instead integrate by parts on (2.33). We get two terms, one from the $r=b$ boundary and the other from precisely the past and future Rindler horizons on the boundary of the domain of dependence of $A_{0}$. It turns out the former term cancels (2.26) prior to taking the $b \rightarrow 0$ limit (although we always need $b$ small), and the later term is exactly the desired result given in (2.27) . So in the end when we add all the terms together, no $b \rightarrow 0$ limit is necessary and the null

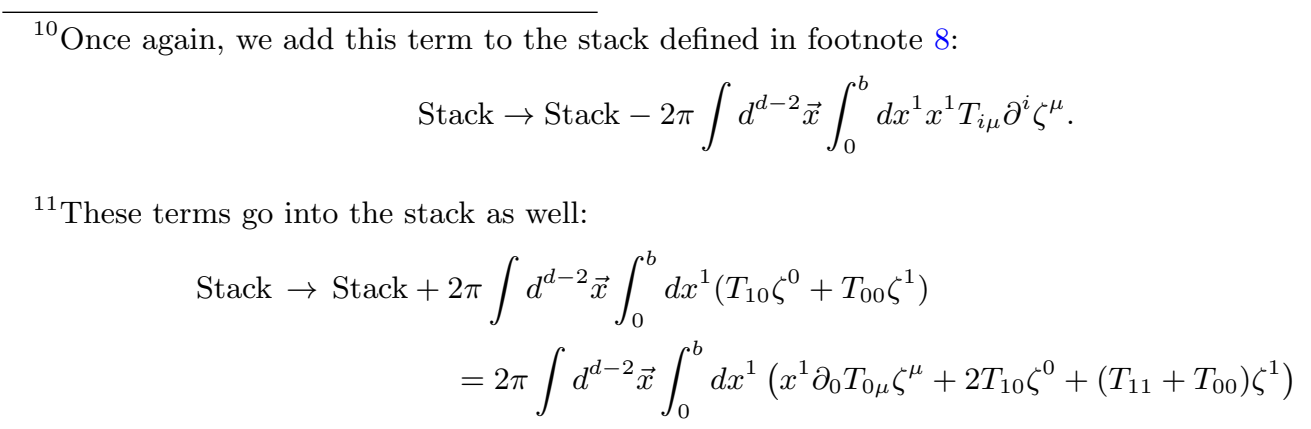

where in the second equality we have integrated by parts; this is then exactly the extension of the $x^{1}$ integral in (2.29) so that it ranges from 0 to $\infty$. Even though all these terms vanish as $b \rightarrow 0$, it is satisfying that they add up like this. 


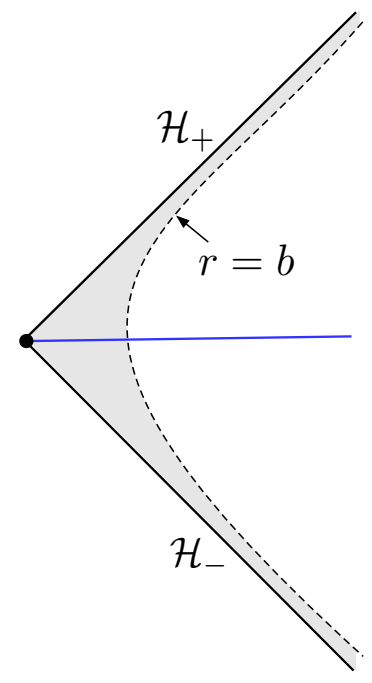

Figure 4. The contribution from inside the region $R_{b}$ can be written in real time as an integral over the shaded region.

energy operators in (2.27) simply emerge. This is perhaps not too surprising since the $r=b$ surface is imaginary, and there should be no dependence on $b$, however we find the detailed cancelations that occur and the form in (2.33) intriguing (including in the running footnote Stack), perhaps hinting that there is a different way to do this calculation directly in real times.

To summarize, putting everything together, we find that the modular Hamiltonian over the deformed half-space is given by

$$
U K_{A} U^{\dagger}=K_{A_{0}}-2 \pi \int_{\mathcal{H}_{+}} \zeta^{+} T_{++}+2 \pi \int_{\mathcal{H}_{-}} \zeta^{-} T_{--}+2 \pi \int_{A_{0}} t^{\mu} \zeta^{\nu}\left[T_{\mu \nu}, K_{A_{0}}\right]
$$

which is the result claimed in (1.14). ${ }^{12}$ We emphasize once again that the $\zeta^{ \pm}$appearing in the second and third terms above are defined at the entangling surface and in particular do not depend on the null coordinates $x^{ \pm}$along the Rindler horizons. We can also derive a similar expression for the modular Hamiltonian corresponding to the complement $A^{c}$

$$
V K_{A^{c}} V^{\dagger}=K_{A_{0}^{c}}+2 \pi \int_{\mathcal{H}_{+}^{c}} \zeta^{+} T_{++}-2 \pi \int_{\mathcal{H}_{-}^{c}} \zeta^{-} T_{--}+2 \pi \int_{A_{0}^{c}} t^{\mu} \zeta^{\nu}\left[T_{\mu \nu}, K_{A_{0}^{c}}\right]
$$

where $\mathcal{H}_{ \pm}^{c}$ are the Rindler horizons corresponding to the complement $A_{0}^{c}$, and $V: \mathfrak{h}_{A^{c}} \rightarrow \mathfrak{h}_{A_{0}^{c}}$ is a unitary transformation. Finally, putting these together, we obtain the following formula for the full modular Hamiltonian

$$
\mathcal{U} \widehat{K}_{A} \mathcal{U}^{\dagger}=\widehat{K}_{A_{0}}-2 \pi \int_{\mathcal{L}_{+}} \zeta^{+} T_{++}+2 \pi \int_{\mathcal{L}_{-}} \zeta^{-} T_{--}+2 \pi \int_{\Sigma} t^{\mu} \zeta^{\nu}\left[T_{\mu \nu}, \widehat{K}_{A_{0}}\right]
$$

where we have defined the light sheets $\mathcal{L}_{ \pm}=\mathcal{H}_{ \pm} \cup \mathcal{H}_{ \pm}^{c}$, and $\mathcal{U}: \mathfrak{h}_{\Sigma} \rightarrow \mathfrak{h}_{\Sigma}$ is a unitary transformation given by $\mathcal{U}=U \otimes V$.

\footnotetext{
${ }^{12}$ Roughly speaking, the "null-energy" terms measure the amount of modular energy leaving the Rindler wedge, while the commutator term comes from the action of the unitary transformations on the original (undeformed) modular Hamiltonian.
} 


\section{Averaged null energy condition}

In this section, we will consider the expectation value $\left\langle\psi\left|\widehat{K}_{A}\right| \psi\right\rangle$ in the class of states (1.15). We will then use the positivity of the operator $\widehat{K}_{A_{0}}-\widehat{K}_{A}$ to prove the averaged null energy condition within this class.

\subsection{Positivity of $\widehat{K}_{A_{0}}-\widehat{K}_{A}$}

For completeness, we begin with a brief review of the argument that $\widehat{K}_{A_{0}}-\widehat{K}_{A}$ is a positive operator, following [22]. ${ }^{13}$ Consider any two states, which we take here to be the vacuum $|0\rangle$ and a non-trivial pure state $|\psi\rangle$. Given an entangling region $A_{0}$ and the corresponding reduced density matrices $\rho_{A_{0}}$ and $\rho_{A_{0}}^{\psi}$, one defines the relative entropy

$$
\begin{aligned}
& S\left(\rho_{A_{0}}^{\psi} \| \rho_{A_{0}}\right)= \operatorname{Tr}_{A_{0}}\left(\rho_{A_{0}}^{\psi} \ln \rho_{A_{0}}^{\psi}\right)-\operatorname{Tr}_{A_{0}}\left(\rho_{A_{0}}^{\psi} \ln \rho_{A_{0}}\right) \\
&= {\left[\operatorname{Tr}_{A_{0}}\left(\rho_{A_{0}}^{\psi} K_{A_{0}}\right)-\operatorname{Tr}_{A_{0}}\left(\rho_{A_{0}} K_{A_{0}}\right)\right] } \\
& \quad+\left[\operatorname{Tr}_{A_{0}}\left(\rho_{A_{0}}^{\psi} \ln \rho_{A_{0}}^{\psi}\right)-\operatorname{Tr}_{A_{0}}\left(\rho_{A_{0}} \ln \rho_{A_{0}}\right)\right] \\
& \equiv \Delta\left\langle K_{A_{0}}\right\rangle-\Delta S_{\mathrm{EE}}\left[A_{0}\right] .
\end{aligned}
$$

where $K_{A_{0}}$ is the modular Hamiltonian corresponding to the vacuum state over the region $A_{0}$.

Relative entropy has a number of interesting properties. For instance, it is a positive quantity

$$
S\left(\rho_{A_{0}}^{\psi} \| \rho_{A_{0}}\right) \geq 0
$$

Further, if we pick another region $A$ such that $A \subset A_{0}$ (more precisely, if $\mathcal{D}(A) \subset$ $\mathcal{D}\left(A_{0}\right)$, where $\mathcal{D}(A)$ is the domain of dependence of $A$ ) then the monotonicity of relative entropy implies

$$
S\left(\rho_{A}^{\psi} \| \rho_{A}\right) \leq S\left(\rho_{A_{0}}^{\psi} \| \rho_{A_{0}}\right)
$$

Intuitively, the relative entropy measures the distinguishability between two states. From this point of view, the monotonicity property states that the distinguishability between two states decreases as we consider their reduced density matrices over smaller and smaller regions. ${ }^{14}$

From equations (3.2) and (3.4), we obtain

$$
\begin{array}{r}
\Delta\left\langle K_{A}\right\rangle-\Delta\left\langle K_{A_{0}}\right\rangle-\Delta S_{\mathrm{EE}}[A]+\Delta S_{\mathrm{EE}}\left[A_{0}\right] \leq 0 \\
\Delta\left\langle K_{A_{0}^{c}}\right\rangle-\Delta\left\langle K_{A^{c}}\right\rangle-\Delta S_{\mathrm{EE}}\left[A_{0}^{c}\right]+\Delta S_{\mathrm{EE}}\left[A^{c}\right] \leq 0
\end{array}
$$

where all modular Hamiltonians are defined relative to the vacuum. Adding the two inequalities we have

$$
\Delta\left\langle\widehat{K}_{A}\right\rangle-\Delta\left\langle\widehat{K}_{A_{0}}\right\rangle-\Delta S_{\mathrm{EE}}[A]+\Delta S_{\mathrm{EE}}\left[A_{0}\right]-\Delta S_{\mathrm{EE}}\left[A_{0}^{c}\right]+\Delta S_{\mathrm{EE}}\left[A^{c}\right] \leq 0
$$

\footnotetext{
${ }^{13}$ Similar arguments have been used in $[4,50]$. A rigorous proof of the positivity of this operator can also be found in [21] which uses methods of algebraic QFT.

${ }^{14}$ See [51-53] for field theoretic calculations of relative entropy in excited states, using a version of the replica trick.
} 
Now, since all vacuum contributions vanish, we can drop the $\Delta$. (This is because $\widehat{K}_{A}$ annihilates the vacuum for any region $A$ ). This implies

$$
\left\langle\widehat{K}_{A}\right\rangle_{\psi}-\left\langle\widehat{K}_{A_{0}}\right\rangle_{\psi} \leq S_{\mathrm{EE}}[\psi, A]-S_{\mathrm{EE}}\left[\psi, A^{c}\right]-S_{\mathrm{EE}}\left[\psi, A_{0}\right]+S_{\mathrm{EE}}\left[\psi, A_{0}^{c}\right]=0
$$

where the last equality follows from the purity of $|\psi\rangle$. Since this is true for any pure state $|\psi\rangle$, we deduce that $\widehat{K}_{A_{0}}-\widehat{K}_{A}$ is a positive operator.

\subsection{Computing $\left\langle\widehat{K}_{A_{0}}\right\rangle_{\psi}-\left\langle\widehat{\boldsymbol{K}}_{\boldsymbol{A}}\right\rangle_{\psi}$}

We now explicitly compute the expectation value of $\widehat{K}_{A_{0}}-\widehat{K}_{A}$ in the state

$$
|\psi\rangle=e^{-\tau H} \sum_{\alpha} c_{\alpha} \mathcal{O}_{\alpha}(0, \mathbf{x})|0\rangle
$$

where as before we take $A_{0}$ to be the half-space $x^{1}>0$, and $A$ to be the deformed half-space. Using the relation

$$
\left\langle\psi\left|\widehat{K}_{A}\right| \psi\right\rangle=\operatorname{Tr}_{A}\left(\rho_{A}^{\psi} K_{A}\right)-\operatorname{Tr}_{A^{c}}\left(\rho_{A^{c}}^{\psi} K_{A^{c}}\right)
$$

we find

$$
\left\langle\psi\left|\left(\widehat{K}_{A_{0}}-\widehat{K}_{A}\right)\right| \psi\right\rangle=\mathfrak{T}^{(1)}+\mathfrak{T}^{(2)}
$$

where

$$
\begin{aligned}
& \mathfrak{T}^{(1)}=-\operatorname{Tr}_{A_{0}}\left(\delta_{\zeta} \rho_{A_{0}}^{\psi} K_{A_{0}}\right)+\operatorname{Tr}_{A_{0} c}\left(\delta_{\zeta} \rho_{A_{0} c}^{\psi} K_{A_{0}^{c}}\right) \\
& \mathfrak{T}^{(2)}=-\operatorname{Tr}_{A_{0}}\left(\rho_{A_{0}}^{\psi} \delta_{\zeta} K_{A_{0}}\right)+\operatorname{Tr}_{A_{0}{ }^{c}}\left(\rho_{A_{0}}{ }_{c} \delta_{\zeta} K_{A_{0}^{c}}\right)
\end{aligned}
$$

where note that the unitary transformations $U$ and $V$ have dropped out inside the trace. The second term above is straightforward to evaluate from equations (2.35) and (2.36):

$$
\mathfrak{T}^{(2)}=2 \pi \int_{\mathcal{L}_{+}} \zeta^{+}\left\langle T_{++}\right\rangle_{\psi}-2 \pi \int_{\mathcal{L}_{-}} \zeta^{-}\left\langle T_{--}\right\rangle_{\psi}+2 \pi \int_{\Sigma} t^{\mu} \zeta^{\nu}\left\langle\left[\widehat{K}_{A_{0}}, T_{\mu \nu}\right]\right\rangle_{\psi}
$$

where we have defined

$$
\langle Y\rangle_{\psi} \equiv \frac{\langle\psi|Y| \psi\rangle}{\langle\psi \mid \psi\rangle}
$$

The first two terms in (3.14) are the appropriate null energy expectation values (albeit integrated along the transverse $\vec{x}$ directions with arbitrary coefficients $\zeta^{ \pm}(\vec{x})$ ) which enter in the averaged null energy condition. We will see below that the last term in (3.14) is precisely cancelled by a contribution coming from $\mathfrak{T}^{(1)}$.

Consider for instance, the first term in $\mathfrak{T}^{(1)}$; from equations (2.9) and (3.12) we obtain

$$
\operatorname{Tr}_{A_{0}}\left(\delta_{\zeta} \rho_{A_{0}}^{\psi} K_{A_{0}}\right)=\frac{1}{2} \int_{R} \delta g_{\mu \nu}(x)\left\{\frac{\left\langle T^{\mu \nu}(x) K_{A_{0}} X\right\rangle}{\langle X\rangle}-\frac{\left\langle T^{\mu \nu}(x) X\right\rangle\left\langle K_{A_{0}} X\right\rangle}{\langle X\rangle^{2}}\right\}
$$

On the right hand side, the correlators $\langle\cdots\rangle$ indicate Euclidean correlation functions on $\mathbb{R}^{d}$, and recall the notation

$$
X=\sum_{\alpha} \sum_{\alpha^{\prime}} c_{\alpha}^{*} c_{\alpha^{\prime}} \mathcal{O}_{\alpha}^{\dagger}(\tau, \mathbf{x}) \mathcal{O}_{\alpha^{\prime}}(-\tau, \mathbf{x})
$$


Note that the Euclidean correlation function appears naturally from the path integral construction of the deformed density matrix for the excited state - we need only add an insertion of $K_{A_{0}}$ along $A_{0}$ and trace - resulting in the above correlation function. Because of the $K_{A_{0}}$ operator insertion, we should remove an infinitesimal cut running along $A_{0}$ from the region over which we integrate the diffeomorphism $R$. Bearing this in mind, we can integrate by parts in the region $R$ to obtain only a contribution from above and below the $K_{A_{0}}$ operator insertion, yielding a commutator:

$$
\operatorname{Tr}_{A_{0}}\left(\delta_{\zeta} \rho_{A_{0}}^{\psi} K_{A_{0}}\right)=-2 \pi \int_{\Sigma} d^{d-2} \vec{x} \int_{0}^{\infty} d x^{1} \zeta_{\mu} t_{\nu}\left\langle\left[K_{A_{0}}, T^{\mu \nu}\left(0, x^{1}, \vec{x}\right)\right]\right\rangle_{\psi}
$$

where we reiterate that $\zeta$ vanishes at the locations of the operators $\mathcal{O}_{\alpha}$, which allows us to drop the divergence of the stress tensor. For the full modular Hamiltonian we then have:

$$
\mathfrak{T}^{(1)}=-2 \pi \int_{\Sigma} d^{d-2} \vec{x} \int_{-\infty}^{\infty} d x^{1} \zeta_{\mu} t_{\nu}\left\langle\left[\widehat{K}_{A_{0}}, T^{\mu \nu}\left(0, x^{1}, \vec{x}\right)\right]\right\rangle_{\psi}
$$

As promised, this term precisely cancels the last term in equation (3.14). We therefore conclude that

$$
\left\langle\widehat{K}_{A_{0}}\right\rangle_{\psi}-\left\langle\widehat{K}_{A}\right\rangle_{\psi}=2 \pi \int_{\mathcal{L}_{+}} \zeta^{+}\left\langle T_{++}\right\rangle_{\psi}-2 \pi \int_{\mathcal{L}_{-}} \zeta^{-}\left\langle T_{--}\right\rangle_{\psi}
$$

Since $\zeta^{+}>0$ and $\zeta^{-}<0$ by construction (see figure 1), the positivity of the operator $\left(\widehat{K}_{A_{0}}-\widehat{K}_{A}\right)$ leads to the averaged null energy conditions

$$
\begin{aligned}
& \int_{-\infty}^{\infty} d x^{+}\left\langle T_{++}\left(x^{+}, x^{-}=0, \vec{x}\right)\right\rangle_{\psi} \geq 0 \\
& \int_{-\infty}^{\infty} d x^{-}\left\langle T_{--}\left(x^{+}=0, x^{-}, \vec{x}\right)\right\rangle_{\psi} \geq 0
\end{aligned}
$$

This concludes our proof that the monotonicity property of $\widehat{K}_{A}$ implies the ANEC. While we presented the proof above in the context of half-spaces in Minkowski space-time, the above calculation can also be extended in an obvious way to general static bifurcate Killing horizons. In this case we would be studying the modular Hamiltonian for small deformations of the entangling cut away from the bifurcation point in the Hartle-Hawking state. The monotonicity constraint then leads to the ANEC for complete null generators of the Killing horizon.

\section{Modular Hamiltonians in AdS/CFT}

In this section we make a connection between our results and the recently proposed JLMS formula [6]:

$$
K_{A}^{\mathrm{CFT}}=\frac{\text { Area }_{\partial \mathcal{M} \backslash A}}{4 G_{N}}+K_{\mathcal{M}}^{\text {bulk }}+\cdots+O\left(G_{N}\right)
$$

where $A$ denotes the boundary subsystem, and $\mathcal{M}$ denotes the bulk region enclosed by the minimal/extremal Ryu-Takayanagi/HRT $[54,55]$ surface $\partial \mathcal{M} \backslash A$ (which ends on the 
entangling surface $\partial A$, and is homologous to $A$ ). Further, the $\cdots$ denote local terms on the extremal surface, which will not be relevant in the following discussion. The result (4.1) arises as a consequence of the formula for quantum corrections to the Ryu-Takaynagi entropy [56, 57] (see also [58].)

Here, we want to perform a simple consistency check of our formula (2.37) for the full modular Hamiltonian of deformed half-spaces against equation (4.1). In particular, restricting to pure states so that the bulk area operator Area $\partial \mathcal{M} \backslash A$ evaluates the same over $A$ and $A^{c}$, (4.1) allows us to equate the full modular energies between the boundary and the bulk theories

$$
\widehat{K}_{A}^{\mathrm{CFT}}=\widehat{K}_{\mathcal{M}}^{\mathrm{bulk}}+\mathcal{O}\left(G_{N}\right)
$$

where note that the local terms on the extremal surface have also dropped out.

In order to make contact with our previous results, we take $A$ to be a small deformation of the boundary half-space $A_{0}=\left\{x^{1}>0, x^{0}=0\right\}$. If we use the coordinates $\left(z, x^{0}, x^{1}, \vec{x}\right)$ on the Poincaré patch of AdS, with

$$
g_{\mathrm{AdS}}=\frac{d z^{2}-\left(d x^{0}\right)^{2}+\left(d x^{1}\right)^{2}+(d \vec{x})^{2}}{z^{2}},
$$

then the corresponding (undeformed) extremal surface in the bulk is given by the codimension two surface $x^{0}=x^{1}=0$, and we have the corresponding undeformed region $\mathcal{M}_{0}=\left\{x^{1}>0, x^{0}=0\right\}$. To linear order in the CFT shape deformation $\zeta$, we then expect

$$
\left\langle\delta_{\zeta} \widehat{K}_{A_{0}}^{\mathrm{CFT}}\right\rangle_{\psi_{\mathrm{CFT}}}=\left\langle\delta_{\zeta_{\mathrm{bulk}}} \widehat{K}_{\mathcal{M}_{0}}^{\mathrm{bulk}}\right\rangle_{\psi_{\mathrm{bulk}}}+\mathcal{O}\left(G_{N}\right)
$$

where we have evaluated the deformations in the CFT and bulk modular Hamiltonians in an excited boundary state $|\psi\rangle_{\text {CFT }}$ and the dual bulk state $|\psi\rangle_{\text {bulk }}$ respectively. Further, $\zeta_{\text {bulk }}$ is the deformation in the bulk minimal surface as a consequence of the boundary shape deformation, and approaches $\zeta$ in the limit $z \rightarrow 0$; it is fixed away from the boundary by the requirement that the deformed bulk surface remain extremal [55].

For simplicity, we focus on null deformations on the CFT side, i.e. $\zeta^{-}=0$. The left-hand side of equation (4.4) has been computed previously; see equation (3.20). Furthermore, if we view the bulk effective theory as a weakly coupled quantum field theory in background AdS geometry, then we expect that our flat space arguments from the previous sections can be extended to the bulk effective theory - this is because we have a Killing vector field in AdS which generates a boost around the unperturbed extremal surface in the $\left(x^{0}, x^{1}\right)$ plane. The bulk modular Hamiltonian then satisfies a covariant version of (3.20):

$$
\left\langle\delta_{\zeta_{\text {bulk }}} \widehat{K}_{\mathcal{M}}^{\text {bulk }}\right\rangle_{\psi_{\text {bulk }}}=-\int_{\mathcal{L}^{+}\left(\partial \mathcal{M}_{0}\right)} \sqrt{h} \zeta_{\text {bulk }}^{+}(z, \vec{x})\left\langle T_{++}^{\text {bulk }}\left(x^{+}, z, \vec{x}\right)\right\rangle_{\psi_{\text {bulk }}}
$$

where $h_{i j}$ is the induced metric on the undeformed minimal surface $\partial \mathcal{M}_{0}$ :

$$
h=\frac{d z^{2}+(d \vec{x})^{2}}{z^{2}} .
$$


A consequence of JLMS combined with our calculations is therefore:

$$
\begin{aligned}
\int_{\mathcal{L}^{+}\left(\partial A_{0}\right)} d^{d-2} \vec{x} d x^{+} & \zeta^{+}\left\langle\psi_{\mathrm{CFT}}\left|T_{++}^{\mathrm{CFT}}\right| \psi_{\mathrm{CFT}}\right\rangle \\
& =\int_{\mathcal{L}^{+}\left(\partial \mathcal{M}_{0}\right)} \sqrt{h} d^{d-2} \vec{x} d x^{+} d z \zeta_{\text {bulk }}^{+}\left\langle\psi_{\text {bulk }}\left|T_{++}^{\text {bulk }}\right| \psi_{\text {bulk }}\right\rangle
\end{aligned}
$$

Our goal now is to establish this equivalence using the usual rules of AdS/CFT [59-61]. In particular in order to apply the JMLS argument the state under consideration must have a perturbative in $G_{N}$ back-reaction on the bulk AdS space-time and so we will consider boundary states of the form $\left|\psi_{\mathrm{CFT}}\right\rangle=O\left(x_{\psi}\right)|0\rangle_{\mathrm{CFT}}$, where $O$ is a single trace primary scalar operator of dimension $\Delta=\mathcal{O}(1)$ in terms of large- $N$ counting. The operator is inserted at $x_{\psi}:\left(x_{\psi}^{0}=-i \tau, x_{\psi}^{1}=0, \vec{x}_{\psi}=0\right)$. In this case the dual bulk state can be identified as $\left|\psi_{\text {bulk }}\right\rangle=\lim _{z \rightarrow 0} z^{-\Delta} \phi\left(z, x_{\psi}\right)|0\rangle_{\text {AdS }}$ for the corresponding bulk field $\phi$. The equality in (4.6) can already be read off from the work of Hofman and Maldacena [39] but here, for completeness, we will give another derivation.

For a CFT with a weakly coupled Einstein gravity dual in the bulk, the l.h.s. can be computed using Witten diagrams. In particular, we assume that the relevant part of the bulk Lagrangian takes the form:

$$
\mathcal{S}^{\text {bulk }} \sim \int_{\mathrm{AdS}_{d+1}} \sqrt{G}\left[-\frac{1}{G_{N}}\left(R_{G}+\Lambda\right)+\frac{1}{2} G^{\mu \nu} \partial_{\mu} \phi \partial_{\nu} \phi-m_{\Delta}^{2} \phi^{2}\right], m_{\Delta}^{2}=\Delta(\Delta-d)
$$

The leading order Witten diagram contribution to the l.h.s. of (4.6) comes from the coupling between the graviton $\left(h^{\mu \nu}=\delta G^{\mu \nu}\right)$ and the scalar stress tensor:

$$
\mathcal{L}_{\text {int }}^{\text {bulk }}=\sqrt{G} h^{\mu \nu} T_{\mu \nu}^{\text {bulk }}(\phi)
$$

where $T_{\mu \nu}^{\text {bulk }}(\phi)=\partial_{\mu} \phi \partial_{\nu} \phi-\frac{1}{2} G_{\mu \nu} \partial_{\alpha} \phi \partial^{\alpha} \phi+\frac{1}{2} G_{\mu \nu} m_{\Delta}^{2} \phi^{2}$ is the leading order bulk stress tensor in $\mathcal{O}(1 / N)$. We thus have for the integrand:

$$
\begin{aligned}
\left\langle O^{\dagger}\left(x_{\psi}^{\star}\right) T_{++}^{\mathrm{CFT}}(x) O\left(x_{\psi}\right)\right\rangle= & \int d z d^{d} x^{\prime} \sqrt{G} D_{++}^{\mu \nu}\left(z, x^{\prime} ; x\right) \\
& \times\left\{\partial_{\mu} D^{\phi}\left(z, x^{\prime} ; x_{\psi}\right) \partial_{\nu} D^{\phi}\left(z, x^{\prime} ; x_{\psi}^{\star}\right)+\ldots\right\} \\
= & \int d z d^{d} y \sqrt{G} D_{++}^{\mu \nu}\left(z, x^{\prime} ; x\right) T_{\mu \nu}^{\mathrm{bulk}}\left(D^{\phi}\left(z, x^{\prime} ; x_{\psi}\right)\right)
\end{aligned}
$$

where $D_{\alpha \beta}^{\mu \nu}\left(z, x^{\prime} ; x\right)$ and $D^{\phi}\left(z, x^{\prime} ; x_{\psi}\right)$ are the bulk-to-boundary propagators for the graviton and scalar respectively. We identify the products of scalar boundary-to-bulk propagators as giving rise to the expectation value of the bulk stress tensor in the state $|\psi\rangle_{\text {bulk: }}$ :

$$
T^{\text {bulk }}\left(D^{\phi}\left(z, x^{\prime} ; x_{\psi}\right)\right)=\left\langle T_{\mu \nu}^{\text {bulk }}\left(z, x^{\prime}\right)\right\rangle_{\psi_{\text {bulk }}}
$$

To see this, we focus on a particular term $\partial_{\mu} \phi \partial_{\nu} \phi$ in $T_{\mu \nu}^{\text {bulk }}(\phi)$. When viewed as a bulk operator, its expectation value in the bulk state $\left|\psi_{\text {bulk }}\right\rangle$ is given by:

$$
\left\langle\partial_{\mu} \phi\left(z, x^{\prime}\right) \partial_{\nu} \phi\left(z, x^{\prime}\right)\right\rangle_{\psi_{\text {bulk }}}=\lim _{\epsilon, \epsilon^{\prime} \rightarrow 0} \epsilon^{-\Delta} \epsilon^{\prime-\Delta}\left\langle\phi\left(\epsilon, x_{\psi}^{\star}\right) \partial_{\mu} \phi\left(z, x^{\prime}\right) \partial_{\nu} \phi\left(z, x^{\prime}\right) \phi\left(\epsilon, x_{\psi}\right)\right\rangle
$$


The leading order (disconnected) diagram of the bulk 4-point function is given by products of bulk-to-bulk propagator: ${ }^{15}$

$$
\begin{aligned}
\left\langle\phi\left(\epsilon, x_{\psi}^{\star}\right) \partial_{\mu} \phi\left(z, x^{\prime}\right)\right. & \left.\partial_{\nu} \phi\left(z, x^{\prime}\right) \phi\left(\epsilon, x_{\psi}\right)\right\rangle \\
& =\partial_{\mu}\left\langle\phi\left(\epsilon, x_{\psi}^{\star}\right) \phi\left(z, x^{\prime}\right)\right\rangle \partial_{\nu}\left\langle\phi\left(z, x^{\prime}\right) \phi\left(\epsilon^{\prime}, x_{\psi}\right)\right\rangle \\
& =\partial_{\mu} D_{\text {bulk-to-bulk }}^{\phi}\left(\epsilon, x_{\psi}^{\star} ; z, x^{\prime}\right) \partial_{\nu} D_{\text {bulk-to-bulk }}^{\phi}\left(z, x^{\prime} ; \epsilon^{\prime}, x_{\psi}\right)
\end{aligned}
$$

The boundary-to-bulk and bulk-to-bulk propagators are related by the limit:

$$
D^{\phi}\left(z, x^{\prime} ; x\right)=\lim _{\epsilon \rightarrow 0} \epsilon^{-\Delta} D_{\text {bulk-to-bulk }}^{\phi}\left(z, x^{\prime} ; \epsilon, x\right)
$$

We thus see that $\partial_{\mu} D^{\phi}\left(z, x^{\prime} ; x_{\psi}^{\star}\right) \partial_{\nu} D^{\phi}\left(z, x^{\prime} ; x_{\psi}\right)=\left\langle\partial_{\mu} \phi\left(z, x^{\prime}\right) \partial_{\nu} \phi\left(z, x^{\prime}\right)\right\rangle_{\psi_{\text {bulk }}}$. Similar relations hold for the other two terms in $T_{\mu \nu}^{\text {bulk }}$ and we conclude that:

$$
\left\langle O^{\dagger}\left(x_{\psi}^{\star}\right) T_{++}^{\mathrm{CFT}}\left(x^{+}, x^{i}\right) O\left(x_{\psi}\right)\right\rangle=\int d z d^{d} x^{\prime} \sqrt{G} D_{++}^{\mu \nu}\left(z, x^{\prime} ; x\right)\left\langle T_{\mu \nu}^{\mathrm{bulk}}\left(z, x^{\prime}\right)\right\rangle_{\psi_{\text {bulk }}}
$$

We need to integrate this relation over $\int d x^{+} d^{d-2} \vec{x} \zeta^{+}\left(x^{i}\right)$ on the boundary. In particular, since $\zeta^{+}(\vec{x})$ is independent of $x^{+}$, we can take it out of the null integral, and replace $\int d x^{+} D_{++}^{\mu \nu}\left(z, x^{\prime} ; x^{+}, \vec{x}\right)=\delta_{++}^{\mu \nu} D^{\text {shock }}\left(z, \vec{x}^{\prime} ; \vec{x}\right) \delta\left(x^{\prime-}\right)$, where $D^{\text {shock }}\left(z, \vec{x}^{\prime} ; \vec{x}\right)$ is the boundaryto-bulk propagator for the shock wave graviton mode: $h^{++}\left(z, x^{\prime-}, \vec{x}\right)=f\left(z, \vec{x}^{\prime}\right) \delta\left(x^{\prime-}\right)$. In $\operatorname{AdS}_{d+1}, D^{\text {shock }}\left(z, \vec{x}^{\prime} ; \vec{x}\right)$ is determined by solving Einstein's equations for this metric fluctuation giving the shock-wave equation: ${ }^{16}$

$$
\left(\partial_{z}^{2}+\partial_{i}^{2}-\frac{d+3}{z} \partial_{z}+\frac{2 d+4}{z^{2}}\right) D^{\text {shock }}\left(z, y^{i} ; x^{i}\right)=0, \quad \lim _{\epsilon \rightarrow 0} D^{\text {shock }}\left(\epsilon, y^{i} ; x^{i}\right) \rightarrow \epsilon^{2} \delta^{d-2}\left(x^{i}-y^{i}\right)
$$

The factor $\delta_{++}^{\mu \nu} \delta\left(x^{\prime-}\right)$ localizes the bulk integral onto $\mathcal{L}^{+}\left(\partial \mathcal{M}_{0}\right)$, and projects onto the $(++)$ component of bulk stress tensor:

$$
\begin{aligned}
\int_{\mathcal{L}^{+}\left(\partial A_{0}\right)} \zeta^{+}\left(x^{i}\right)\left\langle T_{++}^{\mathrm{CFT}}\left(x^{+}, x^{i}\right)\right\rangle_{\psi_{\mathrm{CFT}}} & =\int_{\mathcal{L}^{+}\left(\partial \mathcal{M}_{0}\right)} \sqrt{h} \tilde{\zeta}^{+}\left(z, \vec{x}^{\prime}\right)\left\langle T_{++}^{\text {bulk }}\left(z, x^{\prime-}=0, x^{\prime+}, \vec{x}^{\prime}\right)\right\rangle_{\psi_{\text {bulk }}} \\
\tilde{\zeta}^{+}\left(z, \vec{x}^{\prime}\right) & =z^{-2} \int d^{d-2} \vec{x} \zeta^{+}(\vec{x}) D^{\text {shock }}\left(z, \vec{x}^{\prime} ; \vec{x}\right)
\end{aligned}
$$

One can finally check from (4.15) that $\tilde{\zeta}^{+}\left(z, \vec{x}^{\prime}\right)$ satisfies the extremal bulk extension of $\zeta^{+}(\vec{x})$ on $\partial \mathcal{M}\left(A_{0}\right)$ :

$$
\left(-\frac{d-1}{z} \partial_{z}+\partial_{z}^{2}+\partial_{\vec{x}^{\prime}}^{2}\right) \tilde{\zeta}^{+}\left(z, \vec{x}^{\prime}\right)=0, \lim _{\epsilon \rightarrow 0} \tilde{\zeta}^{+}\left(\epsilon, \vec{x}^{\prime}\right) \rightarrow \zeta^{+}\left(\vec{x}^{\prime}\right)
$$

which is precisely what defines $\zeta_{\text {bulk }}^{+}(z, \vec{x})$, making (4.16) equivalent to (4.6), consistent with JLMS formula.

\footnotetext{
${ }^{15}$ We analytically continue these propagators to real time such that the ordering is the appropriate one for computing expectation values in the state $|\psi\rangle_{\text {bulk }}$.

${ }^{16}$ This shock wave metric is actually a full non-linear solution to Einstein's equations although we have not used this fact.
} 


\section{Discussion}

In this paper, following the circle of ideas in $[16,22,50]$, we established a relation between the monotonicity of relative entropy and the averaged null energy condition in arbitrary QFTs, and in so doing proved the most general Hofman-Maldacena bounds on the data in CFT three-point functions. We will now summarize the perturbative calculation we performed to establish this connection and then conclude with possible future work.

The general goal was to study perturbatively the shape dependence of modular Hamiltonians/energies. We did this by applying "perturbation theory for reduced density matrices" which turns out to have some novel features which we describe now. Schematically the important term in our calculation (2.15) came from expanding the log used to define the modular Hamiltonian. Here we give an alternative description of this expansion:

$$
-\ln \rho_{A_{0}}\left(1+\rho_{A_{0}}^{-1} \delta \rho\right)=K_{A_{0}}-\sum_{n=0}^{\infty}(-1)^{n} \frac{B_{n}}{n !} \underbrace{\left[K_{A_{0}},\left[K_{A_{0}}, \ldots\left[K_{A_{0}}, \rho_{A_{0}}^{-1} \delta \rho\right]\right]\right]}_{n \text {-times }}+O\left(\delta \rho^{2}\right)
$$

where $B_{n}$ are the Bernoulli numbers. The right hand side comes about due to the non commutativity of the two matrices in the log on the left hand side. That is, these are the usual nested commutator terms in the Baker-Campbell-Hausdorff formula keeping only terms to order $\mathcal{O}(\delta \rho)$ (see also [62] for related discussion).

This set of nested commutators clearly has something to do with the evolution with respect to $K_{A_{0}}$ - or in other words modular flow. So it should come as no surprise that these terms can be re-summed into an integral over $\rho_{A_{0}}^{-i s / 2 \pi}\left(\rho_{A_{0}}^{-1} \delta \rho\right) \rho_{A_{0}}^{i s / 2 \pi}$ multiplied by some kernel - a fact we used in (2.17). In fact, in going from equation (5.1) to (2.17), one simply uses the following integral representation of the Bernoulli numbers [63, 64]:17

$$
B_{n}=-\frac{(-i)^{n}}{(2 \pi)^{n}} \int_{-\infty}^{\infty} d s \frac{s^{n}}{4 \sinh ^{2}\left(\frac{s+i \epsilon}{2}\right)} \quad \cdots \quad(n \in \mathbb{Z})
$$

Surprisingly this integral and kernel as well have the effect of switching the original Euclidean diffeomorphism contained in $\delta \rho$ and used to move around the entangling surface, to a real time diffeomorphism determined by the new vector field given in (2.33). From here the null energy operators involved in the ANEC just pop out as boundary terms when integrating by parts over the real time diffeomorphism. Of course in real times now a new boundary has opened up; what previously was the co-dimension 2 entangling surface at the origin in Euclidean space becomes a null hypersurface along the Rindler horizon where the null energy operators are defined.

The non-commutativity emphasized in (5.1) was of fundamental importance to our calculation. We feel that we do not fully understand the magic behind this calculation and that there are new surprises lurking if we go to higher orders in perturbation theory and try to systematize this approach. Similar tools were applied in $[7,12]$ to entanglement entropy

\footnotetext{
${ }^{17}$ Note that we pick the convention where $B_{1}=+\frac{1}{2}$; also recall that $B_{2 m+1}=0$ for $m=1,2 \cdots$. The corresponding terms in the integral representation (5.2) pick out the residue at $s=0$, which is only non-trivial for $n=1$.
} 
where it was important to control these commutator terms in order to find agreement between this perturbative approach and known results from AdS/CFT. Here we have also established a similar agreement with AdS/CFT and in particular the recent proposal by JLMS [6] for the modular Hamiltonian in AdS/CFT.

Apart from gaining a deeper understanding into the inner working of these calculations we now give some detail of future work that we think would be valuable to pursue.

\subsection{Sharpening the argument}

In the main sections of the paper our derivation eschewed any issues related to the precise definition of entanglement and modular energy in quantum field theory. Indeed these quantities are expected to be afflicted by significant UV divergences, and possibly even ambiguities related to how one splits the degrees of freedom between $A$ and $A^{c}$. Thus in order to calculate these quantities we must specify a regulator and a prescription for splitting the Hilbert space. However it became clear to us that we never needed to do this, and so any real discussion of a regulator was relegated to appendix A.

Ultimately this should not have come as a surprise, the final goal was to calculate either relative entropy or the full modular Hamiltonian - both of which are expected to be UV finite quantities and both of which can actually be given a definition directly in the continuum $[47,48]$. This definition however was not convenient for our current calculation so at an intermediate step we needed to calculate the expectation of the full modular Hamiltonian in terms of the UV sensitive (half) modular Hamiltonian. Since we never explicitly saw these UV divergences, our manipulations should be regarded as formal. ${ }^{18}$ Appendix A is an attempt to remedy this, by giving some details of a brick wall like regulator [66] that renders the modular energy and associated quantities well-defined. The brick wall regulator introduces dependence on the boundary conditions one chooses for fields at the wall close to the entangling surface.

The regulated version of relative entropy does not satisfy the property of monotonicity (for a finite but small cutoff scale) since the brick wall cutoff is a rather drastic modification to the theory that does not allow one to compare different spatial regions with the same modification. So to claim a completely rigorous proof of the ANEC we still need to show that when we remove the brick wall cutoff the quantity we get is the continuum version of relative entropy - which is then known to be monotonic [47]. This requires methods that are beyond the scope of this paper, and we leave this to future investigations. Ultimately we would like a mathematically rigorous derivation, perhaps without reference to density matrices and using methods of algebraic quantum field theory [21, 48].

Finally we would like to understand if there are any restrictions on the state in which we calculate the expectation value of the deformed modular Hamiltonian. For example we formulated our state in terms of a local operator insertion at $x_{E}^{0}= \pm \tau$, which is sufficiently general for a CFT. More generally, say for relativistic theories, our argument will go through relatively unmodified if we just insert a general state of the theory and

\footnotetext{
${ }^{18}$ They might be regarded as about as formal as the usual derivation of the replica trick for Renyi entropies in terms of a partition function on a singular surface [65].
} 
its conjugate in flat space along the Euclidean time slices $x_{E}^{0}= \pm \tau$. However we are required to separate the diffeomorphism that moves around the entangling surface away from $\left|x_{E}^{0}\right| \geq \tau$. We can make the region in which the diffeomorphism acts small but we should be limited by $|\zeta|$ the size of the diffeomorphism vector field at the entangling surface. This presumably puts some restriction on the state such that the expectation value of the stress tensor cannot get arbitrarily large. For example if we work with the state created by a local operator insertion $\left|\int_{\mathcal{H}_{ \pm}}\left\langle T_{ \pm \pm}\right\rangle_{\psi}\right| \sim \tau^{-1}<|\zeta|^{-1}$. This is likely just the restriction that the perturbative expansion converge and we can always arrange this to happen by taking a small enough spatial deformation.

\subsection{Generalizations}

One obvious generalization involves attempting to prove the ANEC in other space-times as well as along more general complete achronal null geodesics. ${ }^{19}$ Along these lines it might be an easier first step to try to apply the methods of this paper to stationary but not static black holes with the null generator lying along a bifurcate Killing horizon (like the Kerr black hole). Since we used the framework of perturbation theory starting from a state described by a known density matrix (the Hartle-Hawking state) we are not very optimistic this will succeed when we don't have such a starting point.

Instead perhaps a more fruitful direction to pursue would be to consider the generalized second law (GSL) for quantum fields outside of a black hole with a static bifurcate Killing horizon. Here we are referring to the work of [16] where the GSL was proven for free as well as super renormalizable QFTs. ${ }^{20}$ The GSL applies to the following generalized entropy:

$$
S_{\text {gen }}=\frac{\operatorname{Area}(\partial A)}{4 G_{N}}+S_{\mathrm{EE}}\left(\rho_{A}^{\psi}\right)
$$

where Area $(\partial A)$ refers to the area of a codimension-1 slice of the Killing horizon where the spatial region $A$ ends $(\partial A)$ and $S_{\mathrm{EE}}$ is the entanglement entropy of the quantum fields outside this horizon slice. Applying the monotonicty of relative entropy to $S_{\mathrm{EE}}\left(\rho_{A}^{\psi}\right)$ one finds:

$$
\Delta S_{\text {gen }} \geq \frac{\Delta \operatorname{Area}(\partial A)}{4 G_{N}}+\Delta\left\langle K_{A}\right\rangle_{\psi}
$$

where now $\Delta$ is a finite null deformation $\left(\Delta x^{+}=\zeta^{+}(\vec{y})\right)$ of the entangling surface $\partial A$ to the future of the bifurcation surface $\partial A_{0}$. The change in the area is simply due to the perturbative back reaction of the quantum fields on the space-time via Einstein's equations:

$$
\Delta S_{\mathrm{gen}} \geq 2 \pi \int d^{d-2} \vec{x}\left(-\int_{\zeta^{+}}^{\infty} d x^{+}\left(x^{+}-\zeta^{+}\right)\left\langle T_{++}\right\rangle_{\psi}+\int_{0}^{\infty} d x^{+} x^{+}\left\langle T_{++}\right\rangle_{\psi}\right)+\Delta\left\langle K_{A}\right\rangle_{\psi}
$$

\footnotetext{
${ }^{19}$ These are geodesics where no two points on the curve are timelike separated. The ANEC is known to fail in curved space-times where the null geodesic is chronal [37, 67].

${ }^{20}$ The Hawking area theorem proves the GSL when the area term dominates in the $G_{N} \rightarrow 0$ limit - that is for a classical dynamical background where the classical matter satisfies the NEC. As discussed in [16], what remains, is to prove the GSL when classically the area does not increase - for quantum fields on a stationary black hole background plus free gravitons. For obvious reasons here we then focus on the static case, and leave out gravitons for simplicity.
} 
where we have made use of the Raychaudhuri equation with the correct future boundary condition appropriate for a causal horizon.

To make further progress we need some handle on $K_{A}$ for general null deformations away from $A_{0}$. This does not sound very promising for our perturbative approach, however it does seem like we can carry out our calculations to arbitrary orders in $\zeta^{+}$[68]. Thus with some luck we might be able to prove a statement about $K_{A}$ and get a handle on (5.5) and possibly show the GSL in this case, $\Delta S_{\text {gen }} \geq 0$. A further hint comes actually from AdS/CFT. For a Rindler space cut we have carried out a more detailed calculation ${ }^{21}$ than that outlined in section 4 where we previously showed the equality between the null energy operators in the bulk and boundary. More generally one can show for finite null deformations, but small perturbations to the state (in the $1 / N$ sense):

$$
\begin{aligned}
& 2 \pi \int d^{d-2} \vec{x} \int_{\zeta^{+}}^{\infty} d x^{+}\left(x^{+}-\zeta^{+}\right)\left\langle T_{++}^{\mathrm{CFT}}\right\rangle_{\psi} \\
& =\frac{\operatorname{Area}_{\partial \mathcal{M} \backslash A}(\delta g)}{4 G_{N}}+2 \pi \int d^{d-2} \vec{x} \sqrt{h} \int_{\zeta_{\text {bulk }}^{+}}^{\infty} d x^{+}\left(x^{+}-\zeta_{\text {bulk }}^{+}\right)\left\langle T_{++}^{\text {matter }}\right\rangle_{\psi}
\end{aligned}
$$

and our notation is the same as that in section 4 , where for example $\zeta_{\text {bulk }}^{+}$is the bulk HRT extremal surface corresponding to the deformation $\zeta^{+}$on the boundary and $\mathcal{M}$ is the spatial region between this extremal surface and $A$ on the boundary. Here the area term is the change in the area of the extremal surface due to the backreaction on the metric $\delta g$ in the state $\psi$ (via Einstein's equations.) Note that the extremal surface condition in pure AdS for finite null deformations remains a linear differential equation that matches with the infinitesimal version (4.17) and so $\zeta_{\text {bulk }}^{+}$is the same extension as that used in section 4. Now comparing this statement with that of JLMS [6] we could consistently identify the modular Hamiltonian for finite null deformed regions as:

$$
K_{A}^{\mathrm{CFT}} \stackrel{?}{=} 2 \pi \int d^{d-2} \vec{x} \int_{\zeta^{+}}^{\infty} d x^{+}\left(x^{+}-\zeta^{+}\right) T_{++}^{\mathrm{CFT}}
$$

up to an additive constant, with a similar equation holding for the bulk region modular Hamiltonian $K_{\mathcal{M}}^{\text {bulk }}$. This is certainly not a proof. We have made two guesses (for the bulk and the boundary) and shown them to be self-consistent. And in particular this only works for a special class of states that don't have a large back reaction on the bulk. Note that this last issue also plagued our comparison between the bulk and boundary for small deformations. We simply note here that our perturbative approach, when considered at higher orders, can possibly prove such a statement. ${ }^{22}$ Of course if (5.7) is true then the GSL follows trivially since the right hand side of the inequality in (5.5) just vanishes.

\footnotetext{
${ }^{21}$ This calculation has some overlap with [69] and the details will be reported elsewhere.

${ }^{22}$ Actually a simpler argument is to take the perturbative result we have derived for null shape deformations and then use the QFT boost generator around the original undeformed Rindler cut to amplify the deformation. This boost will then act on the state. However if we work in a sufficiently general state this should not matter. This process seems to work, and agrees with (5.7), when trying to construct the full modular Hamiltonian and we leave the details of how this works for the half space modular Hamiltonian for the future. We thank Aron Wall for suggesting this argument to us.
} 
Finally we point out that in some sense these calculations have already been pushed to higher orders. Rather than consider the excited state modular energy, if we just calculate the modular Hamiltonian in the original vacuum state it should reproduce the entropy of the vacuum. At first order this vanishes but the second order variation of entropy in a CFT was calculated in [7] using similar methods to this paper. This quantity is sometimes referred to as entanglement density [70]. Although it was not realized at the time the answer in [7] can be related to a correlation function of two "null energy operators" - the same null energy operators that appear in the (half sided) modular Hamiltonian in this work. This will be the subject of a forthcoming paper [71]. Taken together this hints at a unifying picture for vacuum entanglement in CFTs related to null energy operators that may even pave the way to a new understanding and proof of the Ryu-Takayanagi [54] and HRT [55] proposals for calculating entanglement entropy in the vacuum state of holographic CFTs.

\section{Acknowledgments}

It is a pleasure to thank Xi Dong, Gary Horowitz, Veronika Hubeny, Aitor Lewkowycz, Don Marolf, Mukund Rangamani, David Simmons-Duffin and Aron Wall for discussions and suggestions. Work supported in part by the U.S. Department of Energy contract DE-FG02-13ER42001 and DARPA YFA contract D15AP00108.

\section{A Cutoff at the entangling surface}

In this appendix we would like to give a prescription for regulating the modular energy that we calculate in the main part of the paper. We go through this in some depth because the arguments we gave previously were somewhat formal. Although the quantity in which we are ultimately interested — the full modular Hamiltonian — is UV finite [48], at intermediate steps we encountered quantities which are not. In particular the modular energy of some state is expected to have the same UV divergences as the entanglement entropy of that state because the difference between them is the relative entropy which is UV finite. ${ }^{23}$ Thus the issues here are the same as the usual issues of defining entanglement entropy in the continuum. ${ }^{24}$ There are several ways to define a regulated version of entanglement entropy, but the most convenient for us will be a "brick wall" regulator [66]. This is so we can still use Euclidean path integral methods to construct the density matrices in question. Apart from possible IR issues the entropies are now finite - the IR issues do not concern us and cancel when evaluating the differences between excited and vacuum states, at least for states that are sufficiently close to the vacuum near the boundaries of space.

\footnotetext{
${ }^{23}$ There are still several reasons to expect some of our intermediate steps to be finite. Any divergences should be local to the entangling surface, and assuming our regulator is geometric[72-74] no such term which respects the $S(A)=S\left(A^{c}\right)$ purity condition can generate a divergence for first order spatial shape deformations. Similarly there is a general expectation that any such divergences cancel in the difference $S\left(\rho_{A}^{\psi}\right)-S\left(\rho_{A}\right)$ although we will find evidence that this cancellation might not always occur. Of course these variations and differences are still calculated in terms of divergent quantities so we proceed.

${ }^{24}$ For a recent discussion of some of the issues involved see $[72,75]$. When the QFT in question is a gauge theory there are even questions about how the degrees of freedom are split between two spatial regions [76].
} 


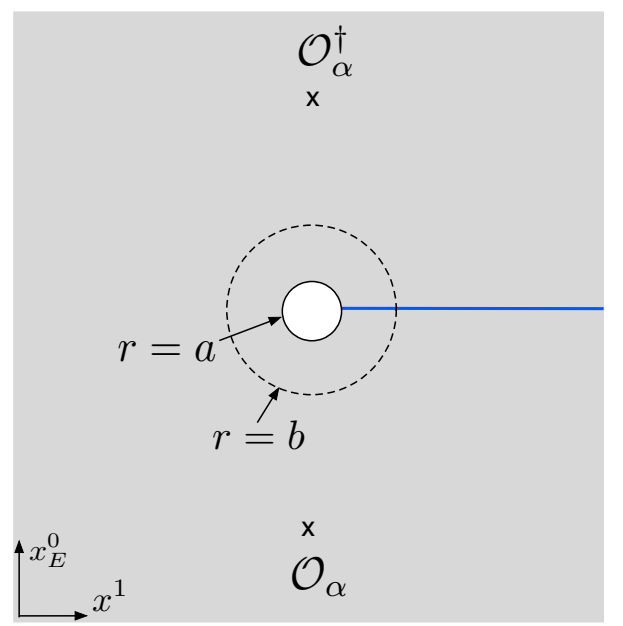

Figure 5. The path integral construction of the regulated reduced density matrix for an excited state. We cut out a cylindrical region of radius $r=a$ around the entangling surface, with brickwall-like boundary conditions. Also shown is the fictitious cutoff surface of radius $r=b$.

Roughly speaking we can simply go through our calculation in sections 2 and 3.2 with the reduced density matrices defined via path integrals on manifolds with a cylindrical region of radius $a$ cut out from around the entangling surface: $\rho_{A_{0}, g} \rightarrow \rho_{A_{0}, g}(a)$ (see figure 2). In order to to do this consistently we should impose boundary conditions on the cutoff surface - we will assume that the boundary conditions at the cutoff surface decouple in the limit $a \rightarrow 0$. We might also need to add new degrees of freedom here $[77,78]$ and there are good reasons to believe these should decouple when calculating such things as relative entropy [76]. We also require the following:

- Rotation/Boost invariance for the undeformed Rindler region. This is so that $K_{A_{0}}$ still has the interpretation as the generator of rotations/boosts around the cutoff surface. For example this will require that the stress tensor at the cutoff surface is constrained to have zero rotation flux $\left.T_{\theta r}\right|_{r \rightarrow a} \rightarrow 0$ into the cutoff cylinder. This should be required as part of the boundary conditions. ${ }^{25}$

- For a more general region - we cut out a cylinder in Gaussian normal coordinates. Here of course we do not have rotation invariance. We use normal coordinates so we can still use the relation (2.5) derived in the main text. One way to do this is to pick the diffeomorphism to map the deformed entangling surface to Gaussian normal coordinates - where the regulator is then picked to be a metric distance $a$ orthogonal to the surface away from $A$. For us this amounts to the choices:

$$
\begin{gathered}
\zeta=\zeta^{z} \partial_{z}+\zeta^{\bar{z}} \partial_{\bar{z}}+\frac{1}{2} \partial^{i}\left(\zeta^{z}\right) \bar{z} \partial_{i}+\frac{1}{2} \partial^{i}\left(\zeta^{\bar{z}}\right) z \partial_{i}+\ldots \\
g_{\mu \nu} d x^{\mu} d x^{\nu}=-d z d \bar{z}+\left(\delta_{i j}+\bar{z} \partial_{i} \partial_{j} \zeta^{z}+z \partial_{i} \partial_{j} \zeta^{\bar{z}}\right) d x^{i} d x^{j}+\ldots
\end{gathered}
$$

\footnotetext{
${ }^{25}$ Note however that this can fail in the case of chiral theories, in which case the boost symmetry is anomalous [79-82].
} 
where we have expanded the diffeomorphism and the metric close to the entangling surface. We then cutoff the path integral which defines $\rho_{A_{0}, g}(a)$ in (2.5) at $r=|z|=a$ supplying some appropriate yet unspecified boundary conditions. After making this slight modifications the diffeomorphism acts the same way as in the bulk of the text in particular there is no boundary term due to the displacement of the cutoff surface (although of course the new stress tensor could have delta function contributions on the cutoff surface.)

- At the very minimum we require that for some local operator inserted in the path integral that defined $\rho_{A_{0}, g}(a)$ we should have:

$$
\lim _{a \rightarrow 0} \operatorname{Tr}_{A_{0}} \rho_{A_{0}, g}^{\psi}(a) \mathcal{O}(x)=\langle\mathcal{O}(x)\rangle_{\psi}
$$

Following the steps below (2.18) in section 2.2 for the change in modular Hamiltonian due to the stress tensor deformation, the differences are due to a slightly modified diffeomorphism and a different region of integration for the stress tensor $R_{0}$ in the Euclidean plane which is cutoff by $r>a$; see figure 2. This cutoff is distinct from the imaginary cutoff surface defined in section 2.2 with $r>b$ and we will take $a \ll b$. Indeed splitting the contribution from the stress tensor integral into the three regions as we did previously, there is only one term which is sensitive to the cutoff in the limit $a \ll b$ and this is the contribution from inside the hole $a<r<b$ which we call $\delta_{\zeta} K_{b}(a)$. The other two contributions from the branch cut and the imaginary cutoff surface give identical results as in the main text. With the same set of manipulations we can write the potentially problematic term as:

$$
\delta_{\zeta} K_{b}(a)=2 \pi \int_{a<r<b} d^{d} x: T_{\mu \nu}: \delta \widetilde{g}^{\mu \nu}
$$

where the resulting real time metric deformation is:

$$
\delta \widetilde{g}_{\mu \nu} d x^{\mu} d x^{\nu}=\frac{1}{2}\left(\Theta\left(x^{0}\right) x^{-} \partial_{i} \partial_{j} \zeta^{+}+\Theta\left(-x^{0}\right) x^{+} \partial_{i} \partial_{j} \zeta^{-}\right) d x^{i} d x^{j}
$$

Note the integration region is now a section of a solid hyperboloid. This is slightly modified from (2.33) and (2.34) since we are now working in Gaussian normal coordinates. Of course to analyze the limiting properties of (A.4) we should trace it against our state $\operatorname{Tr} \rho_{A_{0}}^{\psi}(\cdot)$.

At this point it is possible to remove the brick wall regulator $a \rightarrow 0$ from (A.4). Dependence on $a$ appears in the integration region $R_{0}$ as well as implicitly in $T_{\mu \nu}$ since this is the appropriate field theory stress tensor in the presence of a boundary. Note that the boundary conditions on the brick wall in Euclidean space have naturally been mapped to Rindler space in real times along the hyperbola $r=a,-\infty<s<\infty$. The claim is that we can remove the regulator from the integrand using the requirement (A.3). Of course the remaining $d^{d} x$ integral may still be divergent, however we found this not to be the case in the main text. It is this sense in which we expect the boundary conditions on the surface $r=a$ to decouple. To show this rigorously we would have to show the integrand converges sufficiently uniformly to the $a=0$ limit. Note that the metric deformation $\delta g_{i j} \sim r e^{-|s|}$ in Rindler coordinates and so this is a mild condition on the behavior of the stress tensor 
close to the brick wall. ${ }^{26}$ To say anything further we would need to specify more about the boundary conditions than we are willing to. However since the details of the boundary conditions are not important for defining relative entropy or the full modular Hamiltonian in the continuum of a QFT, it must be the case that any divergences we might see here should cancel when calculating these final quantities. Instead we can turn this condition around and demand that this should be true for any brick wall regulator that is supposed to be a good regulator for calculating modular energy.

We now turn to the second contribution to the deformed modular energy (the first term in (3.12).) Compared to our previously obtained expressions we now find a contribution from the boundary of the cutoff region at $r=a$ which looks like:

$$
\left.\operatorname{Tr}_{A_{0}}\left(\delta_{\zeta} \rho_{A_{0}}^{\psi} K_{A_{0}}\right)\right|_{\partial R_{a}}=a \oint_{\partial R_{a}} \zeta_{\mu} n_{\nu}\left(\left\langle T^{\mu \nu}(x) K_{A_{0}}\right\rangle_{\psi}-\left\langle T^{\mu \nu}(x)\right\rangle_{\psi}\left\langle K_{A_{0}}\right\rangle_{\psi}\right)
$$

If we instead calculate this contribution to the shape deformation of the full modular hamiltonian (this was defined as $\mathfrak{T}^{(1)}$ in $(3.12)$ ) we get a term coming from the complement $A_{0}^{c}$ which adds to give the total contribution to $\mathfrak{T}^{(1)}$ coming from the cutoff surface $\partial R_{a}$ :

$$
\left.\mathfrak{T}^{(1)}\right|_{\partial R_{a}}=-a \oint_{\partial R_{a}} \zeta_{\mu} n_{\nu}\left(\left\langle T^{\mu \nu}(x) \widehat{K}_{A_{0}}\right\rangle_{\psi}-\left\langle T^{\mu \nu}(x)\right\rangle_{\psi}\left\langle\widehat{K}_{A_{0}}\right\rangle_{\psi}\right)
$$

where we remind the reader that $\widehat{K}_{A_{0}}$ is the undeformed full modular Hamiltonian. In the limit $a \rightarrow 0$, the above term appears to be linearly suppressed; however one might worry that there are potential enhancements from the stress tensor coming close to $\widehat{K}_{A_{0}}$ in the first term above. To see that this does not happen, recall that $\widehat{K}_{A_{0}}$ is a conserved charge, namely the generator of rotations around the entangling surface. Consequently, we can freely move it away from cutoff surface as well as the other stress tensor inside the above correlator. Here we have to take into account the fact that the boundary condition on the cutoff surface should not allow for any $\widehat{K}_{A_{0}}$ flux into the cutoff surface $T_{r \theta} \rightarrow 0$. If we could move $\widehat{K}_{A_{0}}$ off to $x_{E}^{0}= \pm \infty$, then the corresponding term would vanish, because $\widehat{K}_{A_{0}}$ annihilates the vacuum. However, as we keep moving $\widehat{K}_{A_{0}}$ away from the stress tensor, we will eventually cross the operator insertions $\mathcal{O}_{\alpha}$ or $\mathcal{O}_{\alpha}^{\dagger}$ (depending on whether we move $\widehat{K}_{A_{0}}$ towards $x_{E}^{0} \rightarrow-\infty$ or $\left.x_{E}^{0} \rightarrow+\infty\right)$. Every such crossing gives a non-trivial contribution of the form $\left\langle T^{\mu \nu}\left[\widehat{K}_{A_{0}}, \mathcal{O}_{\alpha_{m}}\right] \cdots\right\rangle$, where $\cdots$ denotes the remaining operator insertions. However, it should now be clear that these remaining terms are correlation functions between well-separated operators (as long as $\tau \gg a$ ), and we do not get any enhancement to cancel the factor of $a$. Therefore, we conclude that the contribution from the cutoff surface to $\mathfrak{T}^{(1)}$ vanishes in the limit $a \rightarrow 0$. We claim victory.

Before moving on we note that if we did not do the subtraction that defined the full modular energy, the term (A.6) might still be divergent. We can give the following crude estimate for any such divergence. Note that the half sided modular Hamiltonian, as an

\footnotetext{
${ }^{26}$ Note because of the :: vacuum subtraction for the stress tensor any divergence that might appear exactly at the cutoff surface when $a$ is fixed (say due to an image charge) is state independent and will cancel. The potential divergence we are worried about is in the subsequent limit $a \rightarrow 0$.
} 
integral over the stress tensor, can still be moved around but now it is always anchored to the cutoff surface. We can use this freedom to move the two stress tensors in (A.7) as far apart as possible - on opposite sides of the hole. To get an estimate we now replace the correlation function in the first term of (A.6) with the CFT correlation function without the cutoff surface - on flat Euclidean space. We need to consider the OPE of two stress tensors schematically of the from:

$$
T T \sim \sum_{k, \alpha} C_{\alpha}\left(\delta x^{2}\right)^{-d+\Delta_{\alpha} / 2+k / 2} \partial^{k} \mathcal{O}_{\alpha}
$$

where $\delta x^{2} \sim(a+r)^{2}+\left(\vec{x}-\vec{x}^{\prime}\right)^{2}$ and where $r>a$ refers to the location of the modular Hamiltonian stress tensor. Here $\mathcal{O}_{\alpha}$ are some local primary operators and only scalars can possibly contribute a divergence. Close to the entangling surface for any divergent term we can expand $\zeta^{ \pm}(\vec{x})$ and $\left\langle\mathcal{O}_{\alpha}\left(\vec{x}^{\prime}\right)\right\rangle_{\psi}$ by taking $\vec{x}^{\prime} \sim \vec{x}$. We get some leading term from the unit operator. But there are good reasons this term should vanish. Firstly it is state independent and so should occur for the vacuum state, but there is simply no term we can write down at linear order in $\zeta$ which is local to the entangling surface and has the required rotation/boost invariance around the entangling surface. However now consider a non unit operator, we no longer have rotation around the entangling surface. Then using scale invariance we only expect a divergent contribution to the modular energy of the form:

$$
\begin{aligned}
C_{\alpha} a \int d^{d-2} \vec{x} \int d^{d-2} \overrightarrow{x^{\prime}} \int_{a} d r r & \left(\delta x^{2}\right)^{-d+\Delta / 2+1 / 2}\left\langle\partial_{ \pm} \mathcal{O}_{\alpha}\left(\vec{x}^{\prime}\right)\right\rangle_{\psi} \zeta^{ \pm}(\vec{x}) \\
& \sim a^{2-d+\Delta_{\alpha}} C_{\alpha} \int d^{d-2} \vec{x}\left\langle\partial_{ \pm} \mathcal{O}_{\alpha}(\vec{x})\right\rangle_{\psi} \zeta^{ \pm}(\vec{x})
\end{aligned}
$$

Naively one would have expected that such a contribution is not possible for uniform deformations - $\zeta^{ \pm}$independent of $\vec{x}$ - since in that case we can write an expression for $K_{A}$ and $K_{A_{0}}$ in the absence of the cutoff surface and there is seemingly no divergence. However since these are half sided modular Hamiltonians it seems we should have allowed for the possibility that even the un-deformed $K_{A_{0}}$ has a local divergence:

$$
K_{A_{0}} \sim a^{2-d+\Delta_{\alpha}} C_{\alpha} \int_{\partial A_{0}} \mathcal{O}_{\alpha}+\text { finite }
$$

At least this seems to be required if we want the answer to be consistent with our UV regulator and diffeomorphism invariance. This calculation is far too crude to be trusted, but it does suggest that any kind of brick wall cutoff leaves one susceptible to state dependent divergences in the half sided modular energy of the above nature. In the main text we could have taken such divergences into account simply by adding (A.10) and this would have generated the term in (A.9) without any need for a brick wall.

This new divergent contribution occurs if there is a very relevant $\Delta_{\alpha} \leq d-2$ scalar operator appearing in the TT OPE. For example it cannot be charged under any symmetries. Symmetries would also disallow (A.10). It is not clear a theory with such a scalar operator can exist — see [4] for a related appearance of such operators in state dependent divergences for entanglement entropy. In recent proofs of the Hofman-Maldacena bounds from bootstrap methods, these operators also make an appearance [43]. In our work they are always harmless and cancel when we calculate the full modular Hamiltonian. 
Open Access. This article is distributed under the terms of the Creative Commons Attribution License (CC-BY 4.0), which permits any use, distribution and reproduction in any medium, provided the original author(s) and source are credited.

\section{References}

[1] J.J. Bisognano and E.H. Wichmann, On the duality condition for quantum fields, J. Math. Phys. 17 (1976) 303 [inSPIRE].

[2] H. Casini, M. Huerta and R.C. Myers, Towards a derivation of holographic entanglement entropy, JHEP 05 (2011) 036 [arXiv: 1102.0440] [INSPIRE].

[3] R. Bousso, H. Casini, Z. Fisher and J. Maldacena, Proof of a quantum Bousso bound, Phys. Rev. D 90 (2014) 044002 [arXiv:1404.5635] [InSPIRE].

[4] R. Bousso, H. Casini, Z. Fisher and J. Maldacena, Entropy on a null surface for interacting quantum field theories and the Bousso bound, Phys. Rev. D 91 (2015) 084030 [arXiv: 1406.4545] [INSPIRE].

[5] D.L. Jafferis and S.J. Suh, The gravity duals of modular hamiltonians, arXiv:1412.8465 [INSPIRE].

[6] D.L. Jafferis, A. Lewkowycz, J. Maldacena and S.J. Suh, Relative entropy equals bulk relative entropy, JHEP 06 (2016) 004 [arXiv: 1512.06431] [INSPIRE].

[7] T. Faulkner, R.G. Leigh and O. Parrikar, Shape dependence of entanglement entropy in conformal field theories, JHEP 04 (2016) 088 [arXiv: 1511.05179] [INSPIRE].

[8] V. Rosenhaus and M. Smolkin, Entanglement entropy: a perturbative calculation, JHEP 12 (2014) 179 [arXiv:1403.3733] [INSPIRE].

[9] V. Rosenhaus and M. Smolkin, Entanglement entropy for relevant and geometric perturbations, JHEP 02 (2015) 015 [arXiv: 1410.6530] [INSPIRE].

[10] A. Allais and M. Mezei, Some results on the shape dependence of entanglement and Rényi entropies, Phys. Rev. D 91 (2015) 046002 [arXiv:1407.7249] [INSPIRE].

[11] M. Mezei, Entanglement entropy across a deformed sphere, Phys. Rev. D 91 (2015) 045038 [arXiv: 1411.7011] [INSPIRE].

[12] T. Faulkner, Bulk emergence and the RG flow of entanglement entropy, JHEP 05 (2015) 033 [arXiv: 1412.5648] [INSPIRE].

[13] H. Casini and M. Huerta, A finite entanglement entropy and the c-theorem, Phys. Lett. B 600 (2004) 142 [hep-th/0405111] [INSPIRE].

[14] H. Casini and M. Huerta, On the RG running of the entanglement entropy of a circle, Phys. Rev. D 85 (2012) 125016 [arXiv:1202.5650] [INSPIRE].

[15] H. Casini, Relative entropy and the Bekenstein bound, Class. Quant. Grav. 25 (2008) 205021 [arXiv:0804.2182] [INSPIRE].

[16] A.C. Wall, A proof of the generalized second law for rapidly changing fields and arbitrary horizon slices, Phys. Rev. D 85 (2012) 104049 [arXiv:1105.3445] [INSPIRE].

[17] N. Lashkari, C. Rabideau, P. Sabella-Garnier and M. Van Raamsdonk, Inviolable energy conditions from entanglement inequalities, JHEP 06 (2015) 067 [arXiv:1412.3514] [INSPIRE]. 
[18] N. Lashkari, J. Lin, H. Ooguri, B. Stoica and M. Van Raamsdonk, Gravitational positive energy theorems from information inequalities, arXiv:1605.01075 [INSPIRE].

[19] S. Banerjee, A. Bhattacharyya, A. Kaviraj, K. Sen and A. Sinha, Constraining gravity using entanglement in AdS/CFT, JHEP 05 (2014) 029 [arXiv: 1401.5089] [INSPIRE].

[20] A. Bhattacharyya, L. Cheng and L.-Y. Hung, Relative entropy, mixed gauge-gravitational anomaly and causality, JHEP 07 (2016) 121 [arXiv: 1605.02553] [INSPIRE].

[21] H. Borchers, On the use of modular groups in quantum field theory., Ann. Inst. Henri Poincaré, Phys. Théor. 63 (1995) 331.

[22] D.D. Blanco and H. Casini, Localization of negative energy and the Bekenstein bound, Phys. Rev. Lett. 111 (2013) 221601 [arXiv:1309.1121] [INSPIRE].

[23] A. Borde, Geodesic focusing, energy conditions and singularities, Class. Quant. Grav. 4 (1987) 343 [INSPIRE].

[24] F.J. Tipler, Energy conditions and spacetime singularities, Phys. Rev. D 17 (1978) 2521 [INSPIRE].

[25] J.L. Friedman, K. Schleich and D.M. Witt, Topological censorship, Phys. Rev. Lett. 71 (1993) 1486 [Erratum ibid. 75 (1995) 1872] [gr-qc/9305017] [INSPIRE].

[26] C.J. Fewster, Lectures on quantum energy inequalities, arXiv:1208.5399 [INSPIRE].

[27] R. Bousso, Z. Fisher, S. Leichenauer and A.C. Wall, Quantum focusing conjecture, Phys. Rev. D 93 (2016) 064044 [arXiv:1506.02669] [InSPIRE].

[28] R. Bousso, Z. Fisher, J. Koeller, S. Leichenauer and A.C. Wall, Proof of the quantum null energy condition, Phys. Rev. D 93 (2016) 024017 [arXiv: 1509.02542] [INSPIRE].

[29] J. Koeller and S. Leichenauer, Holographic proof of the quantum null energy condition, Phys. Rev. D 94 (2016) 024026 [arXiv: 1512.06109] [INSPIRE].

[30] G. Klinkhammer, Averaged energy conditions for free scalar fields in flat space-times, Phys. Rev. D 43 (1991) 2542 [INSPIRE].

[31] L.H. Ford and T.A. Roman, Averaged energy conditions and quantum inequalities, Phys. Rev. D 51 (1995) 4277 [gr-qc/9410043] [INSPIRE].

[32] A. Folacci, Averaged null energy condition for electromagnetism in Minkowski space-time, Phys. Rev. D 46 (1992) 2726 [INSPIRE].

[33] R. Verch, The averaged null energy condition for general quantum field theories in two-dimensions, J. Math. Phys. 41 (2000) 206 [math-ph/9904036] [INSPIRE].

[34] W.R. Kelly and A.C. Wall, Holographic proof of the averaged null energy condition, Phys. Rev. D 90 (2014) 106003 [arXiv:1408.3566] [INSPIRE].

[35] D.M. Hofman, Higher derivative gravity, causality and positivity of energy in a UV complete QFT, Nucl. Phys. B $\mathbf{8 2 3}$ (2009) 174 [arXiv:0907.1625] [InSPIRE].

[36] E.E. Flanagan and R.M. Wald, Does back reaction enforce the averaged null energy condition in semiclassical gravity?, Phys. Rev. D 54 (1996) 6233 [gr-qc/9602052] [INSPIRE].

[37] N. Graham and K.D. Olum, Achronal averaged null energy condition, Phys. Rev. D 76 (2007) 064001 [arXiv:0705.3193] [INSPIRE]. 
[38] E.-A. Kontou and K.D. Olum, Proof of the averaged null energy condition in a classical curved spacetime using a null-projected quantum inequality, Phys. Rev. D 92 (2015) 124009 [arXiv: 1507.00297] [INSPIRE].

[39] D.M. Hofman and J. Maldacena, Conformal collider physics: energy and charge correlations, JHEP 05 (2008) 012 [arXiv:0803.1467] [INSPIRE].

[40] M. Kulaxizi and A. Parnachev, Energy flux positivity and unitarity in CFTs, Phys. Rev. Lett. 106 (2011) 011601 [arXiv: 1007.0553] [INSPIRE].

[41] Z. Komargodski, M. Kulaxizi, A. Parnachev and A. Zhiboedov, Conformal field theories and deep inelastic scattering, arXiv:1601.05453 [INSPIRE].

[42] D.M. Hofman, D. Li, D. Meltzer, D. Poland and F. Rejon-Barrera, A proof of the conformal collider bounds, JHEP 06 (2016) 111 [arXiv: 1603.03771] [INSPIRE].

[43] T. Hartman, S. Jain and S. Kundu, A new spin on causality constraints, arXiv:1601.07904 [INSPIRE].

[44] T. Hartman, S. Jain and S. Kundu, Causality constraints in conformal field theory, JHEP 05 (2016) 099 [arXiv : 1509.00014] [INSPIRE].

[45] X.O. Camanho and J.D. Edelstein, Causality constraints in AdS/CFT from conformal collider physics and Gauss-Bonnet gravity, JHEP 04 (2010) 007 [arXiv:0911.3160] [INSPIRE].

[46] A. Buchel, J. Escobedo, R.C. Myers, M.F. Paulos, A. Sinha and M. Smolkin, Holographic GB gravity in arbitrary dimensions, JHEP 03 (2010) 111 [arXiv:0911.4257] [INSPIRE].

[47] H. Araki, Relative entropy of states of von neumann algebras, Publ. Res. Inst. Math. Sci. 11 (1976) 809.

[48] R. Haag, Local quantum physics: fields, particles, algebras, Springer, Germany (2012).

[49] S. Banerjee, Wess-Zumino consistency condition for entanglement entropy, Phys. Rev. Lett. 109 (2012) 010402 [arXiv:1109.5672] [INSPIRE].

[50] A.C. Wall, Proving the achronal averaged null energy condition from the generalized second law, Phys. Rev. D 81 (2010) 024038 [arXiv:0910.5751] [INSPIRE].

[51] N. Lashkari, Relative entropies in conformal field theory, Phys. Rev. Lett. 113 (2014) 051602 [arXiv: 1404.3216] [INSPIRE].

[52] N. Lashkari, Modular Hamiltonian for excited states in conformal field theory, Phys. Rev. Lett. 117 (2016) 041601 [arXiv: 1508.03506] [INSPIRE].

[53] G. Sárosi and T. Ugajin, Relative entropy of excited states in two dimensional conformal field theories, JHEP 07 (2016) 114 [arXiv:1603.03057] [INSPIRE].

[54] S. Ryu and T. Takayanagi, Holographic derivation of entanglement entropy from AdS/CFT, Phys. Rev. Lett. 96 (2006) 181602 [hep-th/0603001] [INSPIRE].

[55] V.E. Hubeny, M. Rangamani and T. Takayanagi, A covariant holographic entanglement entropy proposal, JHEP 07 (2007) 062 [arXiv: 0705.0016] [INSPIRE].

[56] T. Faulkner, A. Lewkowycz and J. Maldacena, Quantum corrections to holographic entanglement entropy, JHEP 11 (2013) 074 [arXiv:1307.2892] [INSPIRE].

[57] N. Engelhardt and A.C. Wall, Quantum extremal surfaces: holographic entanglement entropy beyond the classical regime, JHEP 01 (2015) 073 [arXiv:1408.3203]. 
[58] X. Dong, D. Harlow and A.C. Wall, Reconstruction of bulk operators within the entanglement wedge in gauge-gravity duality, Phys. Rev. Lett. 117 (2016) 021601 [arXiv:1601.05416] [INSPIRE].

[59] J. Maldacena, The large-N limit of superconformal field theories and supergravity, Int. J. Theor. Phys. 38 (1999) 1113 [hep-th/9711200].

[60] E. Witten, Anti-de Sitter space and holography, Adv. Theor. Math. Phys. 2 (1998) 253 [hep-th/9802150] [INSPIRE].

[61] S.S. Gubser, I.R. Klebanov and A.M. Polyakov, Gauge theory correlators from noncritical string theory, Phys. Lett. B 428 (1998) 105 [hep-th/9802109] [INSPIRE].

[62] W.R. Kelly, K. Kuns and D. Marolf, 't Hooft suppression and holographic entropy, JHEP 10 (2015) 059 [arXiv: 1507.03654] [INSPIRE].

[63] NIST Digital Library of Mathematical Functions, http://dlmf.nist.gov/, release 1.0.10 (2015).

[64] F.W.J. Olver eds., NIST handbook of mathematical functions, Cambridge University Press, Cambridge, U.K. (2010).

[65] P. Calabrese and J.L. Cardy, Entanglement entropy and quantum field theory, J. Stat. Mech. 0406 (2004) P06002 [hep-th/0405152] [INSPIRE].

[66] G. Hooft, On the quantum structure of a black hole, Nucl. Phys. B 256 (1985) 727.

[67] M. Visser, Gravitational vacuum polarization. 2: energy conditions in the Boulware vacuum, Phys. Rev. D 54 (1996) 5116 [gr-qc/9604008] [INSPIRE].

[68] T. Faulkner and O. Parrikar, Entanglement entropy and shape perturbation theory, in preparation.

[69] W. Bunting, Z. Fu and D. Marolf, A coarse-grained generalized second law for holographic conformal field theories, Class. Quant. Grav. 33 (2016) 055008 [arXiv:1509.00074] [INSPIRE].

[70] J. Bhattacharya, V.E. Hubeny, M. Rangamani and T. Takayanagi, Entanglement density and gravitational thermodynamics, Phys. Rev. D 91 (2015) 106009 [arXiv:1412.5472] [INSPIRE].

[71] S. Balakrishnan and T. Faulkner, Entanglement density via null energy correlators and gravitational shockwaves, in preparation.

[72] H. Casini, M. Huerta, R.C. Myers and A. Yale, Mutual information and the F-theorem, JHEP 10 (2015) 003 [arXiv: 1506.06195] [INSPIRE].

[73] H. Liu and M. Mezei, A refinement of entanglement entropy and the number of degrees of freedom, JHEP 04 (2013) 062 [arXiv: 1202.2070] [INSPIRE].

[74] T. Grover, A.M. Turner and A. Vishwanath, Entanglement entropy of gapped phases and topological order in three dimensions, Phys. Rev. B 84 (2011) 195120 [arXiv:1108.4038] [INSPIRE].

[75] K. Ohmori and Y. Tachikawa, Physics at the entangling surface, J. Stat. Mech. 1504 (2015) P04010 [arXiv: 1406.4167].

[76] H. Casini, M. Huerta and J.A. Rosabal, Remarks on entanglement entropy for gauge fields, Phys. Rev. D 89 (2014) 085012 [arXiv: 1312.1183] [inSPIRE].

[77] W. Donnelly and A.C. Wall, Entanglement entropy of electromagnetic edge modes, Phys. Rev. Lett. 114 (2015) 111603 [arXiv:1412.1895] [INSPIRE]. 
[78] W. Donnelly and A.C. Wall, Geometric entropy and edge modes of the electromagnetic field, arXiv: 1506.05792 [INSPIRE].

[79] A. Castro, S. Detournay, N. Iqbal and E. Perlmutter, Holographic entanglement entropy and gravitational anomalies, JHEP 07 (2014) 114 [arXiv:1405.2792] [INSPIRE].

[80] N. Iqbal and A.C. Wall, Anomalies of the entanglement entropy in chiral theories, arXiv: 1509.04325 [INSPIRE].

[81] T. Nishioka and A. Yarom, Anomalies and entanglement entropy, JHEP 03 (2016) 077 [arXiv: 1509.04288] [INSPIRE].

[82] T.L. Hughes, R.G. Leigh, O. Parrikar and S.T. Ramamurthy, Entanglement entropy and anomaly inflow, Phys. Rev. D 93 (2016) 065059 [arXiv:1509.04969] [InSPIRE]. 